\title{
MOF Derived Porous ZnO/C Nanocomposites for Efficient Dye Photodegradation
}

\author{
Mian Zahid Hussain, ${ }^{\mathrm{a}, \mathrm{b}}$ Andreas Schneemann, ${ }^{\mathrm{b}, \mathrm{c}}$ Roland A. Fischer, ${ }^{\mathrm{b}}$ Yanqiu Zhu, and Yongde \\ $\mathrm{Xia}^{*}, \mathrm{a}$
}

${ }^{a}$ College of Engineering, Mathematics and Physical Sciences, University of Exeter, Exeter EX4 4QF, U. K.

${ }^{\mathrm{b}}$ Catalysis Research Center, Department of Chemistry, Technical University of Munich, Ernst-OttO-Fischerstraße 1, 85748 Garching, Germany.

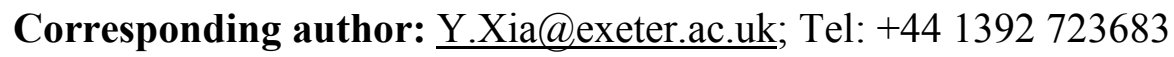




\begin{abstract}
Homogeneously dispersed crystalline $\mathrm{ZnO}$ nanoparticles embedded in porous carbon matrix were synthesized via a one-step carbonization of porous metal-organic framework MOF-5 at 800 ${ }^{\circ} \mathrm{C}$ and $1000{ }^{\circ} \mathrm{C}$ in different gas atmospheres. The resulting $\mathrm{ZnO} / \mathrm{C}$ nanocomposites generally retain cubic particle morphology and high specific surface area of the precursor MOF-5. Various characterization techniques, including XRD, SEM, TEM, elemental mapping, Raman, FTIR and XPS, confirmed that the carbonization of MOF-5 in water vapor atmosphere produced homogeneously dispersed $\mathrm{ZnO}$ nanoparticles confined within the functionalized porous carbon matrix. The rich oxygen-containing hydrophilic functional groups on the surface of the nanocomposite, the defects in the carbon doped $\mathrm{ZnO}$ nanostructure, the crystalline $\mathrm{ZnO}$ nanoparticles and the high textural properties resulted in excellent methylene blue adsorption and photodegradation performance under visible light irradiation. This simple and environmentally friendly approach can be further employed to harvest a variety of new homogeneously dispersed functional metal oxide/carbon composites for various environment and energy related applications.
\end{abstract}

KEYWORDS: Zinc oxide, Metal-organic framework, carbon doping, photocatalysis, dye degradation 


\section{INTRODUCTION}

Due to the high industrialization, fresh water sources and eventually the oceans are polluted every day. Big industries produce a variety of hazardous contaminants, such as organic chemical wastes, byproducts from textile dyes, petrochemicals, pesticides and plastics etc. The treatment of industrial wastewater has become one of the major challenges in our society. There have been enormous efforts to utilize metal oxide as photocatalysts to decompose organic pollutants in wastewater into environmentally benign species. ${ }^{1}$ Zinc oxide $(\mathrm{ZnO})$ is an excellent semiconductor material for this purpose because of its nontoxicity and abundance. ${ }^{2,3}$ However, the wide energy band gap of $\mathrm{ZnO}$ hinders its utilization in the visible light range of the electromagnetic spectrum since bulk $\mathrm{ZnO}$ can only absorbs UV light with wavelength shorter than $380 \mathrm{~nm}$, which consequently restricts the photocatalytic efficiency. ${ }^{4}$ Furthermore, the low surface area of $\mathrm{ZnO}$ and its tendency to agglomerate due to high surface energies are big challenges to effectively utilize $\mathrm{ZnO}$ nanoparticles for photocatalysis. ${ }^{5,6}$

To overcome these limitations, plenty of approaches were developed to generate $\mathrm{ZnO}$ based composites so that the band gap and therefore the photocatalytic performance of the materials can be tuned. ${ }^{7}$ However, the composites synthesized by mechanical and physical methods usually possess low surface area, poor dispersion and heterogeneity. ${ }^{7}$ In the past several years, carbonization of MOFs precursors has drawn huge attention due to the tunable properties of the resulting materials. ${ }^{8}$ Actually, metal-organic frameworks (MOFs) have been proved to be very promising sacrificial templates to generate $\mathrm{ZnO}$ nanaoparticles embedded in porous carbon frameworks with high specific surface areas, tunable pore sizes, increased thermal stability and homogeneously dispersed crystalline $\mathrm{ZnO}$ nanoparticles. ${ }^{9}$ Simultaneously, during the high temperature heat treatment of MOFs, the formed carbon derived from the carbonization of organic linkers also diffuses into the crystal lattice of $\mathrm{ZnO}$ which can adjust the energy band gap of $\mathrm{ZnO} .{ }^{10}$ Consequently, it may result in high performance materials for photocatalytic applications.

MOFs are porous crystalline structures, consisting of metal containing vertices interconnected by organic struts. ${ }^{11-13} \mathrm{In} \mathrm{Zn}$-based MOF-5, the $\mathrm{Zn} 4 \mathrm{O}$ metal cluster is connected to six carboxylates, constructing octahedral-shaped building units linked through rod-like organic $\operatorname{BDC}^{2-}(1,4-b e n z e n e d i c a r b o x y l a t e)$ linkers to form a cubic structure with the composition 
$\mathrm{Zn}_{4} \mathrm{O}(\mathrm{BDC})_{3} \cdot{ }^{14}$ Due to their permanent high porosity, structural flexibility and wide functionalities, MOFs are considered as ideal candidates for applications in gas separation, ${ }^{15}$ sensors, ${ }^{16}$ biomedicine, ${ }^{17}$ super-capacitors ${ }^{18}$, Li-ion batteries ${ }^{19}$ and catalysis. ${ }^{20-21}$ However, the direct employment of MOF-5 in photocatalytic wastewater treatment has not been promising due to the structural instability upon moisture exposure. ${ }^{22}$ To retain the inherent structures, porosity and surface functionalities of MOF-5, one step pyrolysis of MOF-5 was carried out to derive the $\mathrm{ZnO} / \mathrm{C}$ nanocomposites.

The adsorption and photocatalytic degradation of organic dyes depends on several parameters, including the surface charges, surface functionalization, hydrophilic/hydrophobic nature and textural properties of the products. ${ }^{9}$ The open pore networks can facilitate the diffusion of organic dye molecules to access the pore surface lined with $\mathrm{ZnO}$ photocatalysts. Upon visible light irradiation, electron-hole pairs are generated in semiconducting $\mathrm{ZnO} / \mathrm{C}$ nanocomposites. The generated electrons transfer to the conduction band and produce superoxides $\left(\mathrm{O}_{2}^{-}\right)$and the holes in valance band form hydroxyl $(\cdot \mathrm{OH})$ radicals via water oxidation. The reduction of organic dye is induced by superoxides, whereas the $\cdot \mathrm{OH}$ radicals oxidize the organic dye leading to its complete decoloration and finally mineralization. ${ }^{1,23-25}$

A few reports on $\mathrm{MOF}$ derived $\mathrm{ZnO} / \mathrm{C}$ nanocomposites for photocatalytic water treatment have been published. ${ }^{26}$ However, many questions remain unanswered. For instance, the interactions of the nanocomposites with organic dyes and the impact of defects and functional groups on photocatalytic activity have not been thoroughly studied. In 2011, Park et al. prepared $\mathrm{ZnO} / \mathrm{C}$ hybrids by direct carbonization of MOF- 5 at $600{ }^{\circ} \mathrm{C}$ in $\mathrm{N}_{2}$ atmosphere and the resulting $\mathrm{ZnO} / \mathrm{C}$ composite showed photocatalytic Rhodamine $\mathrm{B}$ degradation under UV irradiation. ${ }^{26}$ Recently, we exploited the use of water steam atmosphere to synthesize $\mathrm{ZnO} / \mathrm{N}$-doped nanoporous carbon composites from zeolitic imidazolate framework ZIF-8 $\left(\mathrm{Zn}(\mathrm{mIm})_{2}\right.$; where $\mathrm{mIm}$ is 2-methylimidazole) at $800^{\circ} \mathrm{C}$ for $\mathrm{CO}_{2}$ uptake and organic pollutant methylene blue (MB) removal from water. ${ }^{27}$

In this contribution, the effect of temperatures and the gaseous atmospheres during the carbonization process on the compositions, the morphologies and properties of the resulting $\mathrm{ZnO} / \mathrm{C}$ nanocomposites have been studied. Though there are a few studies available on MOF derived $\mathrm{ZnO} / \mathrm{C}$ composites for photocatalysis, to the best of our knowledge, no detailed study is 
available on the effect of gaseous atmosphere and calcination temperature on the derived $\mathrm{ZnO} / \mathrm{C}$ composites. Therefore, this work provides a novel insight to understand the role of $\mathrm{ZnO}$ and carbon species in MOF-5 derived nanocomposites for the adsorption and photodegradation of MB. To achieve this purpose, we used as-synthesized MOF-5 as precursor to obtain $\mathrm{ZnO} / \mathrm{C}$ at 800 and $1000^{\circ} \mathrm{C}$ under a variety of gaseous atmospheres. In order to control the $\mathrm{C}$ to $\mathrm{ZnO}$ ratios in the nanocomposites with different functional groups, water was introduced as a weak oxidizing agent to conduct a mild oxidation process at high temperatures without compromising the sample morphologies. The controlled introduction of an oxygen source enables the targeted oxidation of carbon and cationic $\mathrm{Zn}^{2+}$, resulting in the formation of homogeneously dispersed porous $\mathrm{ZnO} / \mathrm{C}$ with modified energy band gap. Along with the retained morphology, the formed defects and oxygen-containing functionalities, the obtained $\mathrm{ZnO} / \mathrm{C}$ lead to excellent methylene blue (MB) adsorption and photodegradation performances under visible light irradiation.

Therefore, this work contributes to comprehensive understanding of the behaviors of adsorption and photodegradation of organic dyes on MOF-5 derived $\mathrm{ZnO} / \mathrm{C}$ nanocomposites under visible light irradiation. Based on our study, it can be established that pure carbon sample C-Ar1000 shows only adsorption without any dye degradation under visible light. MOF derived pure $\mathrm{ZnO}$ nanoparticles samples exhibit no adsorption but photodegradation of $\mathrm{MB}$ under visible light. However, the samples prepared under argon saturated water vapors show both adsorption and photodegradation of MB. It is observed that photodegradation of MB is more dominant in $\mathbf{Z n O} / \mathbf{C}-\mathbf{A r}+\mathbf{W}(\mathbf{S})_{800}$ due to the high content of $\mathrm{ZnO}$ whereas adsorption of $\mathrm{MB}$ is dominated in $\mathbf{Z n O / C - A r + W ( S ) 1 0 0 0 ~ d u e ~ t o ~ t h e ~ h i g h ~ l e v e l ~ o f ~ p o r o u s ~ c a r b o n . ~}$

\section{EXPERIMENTAL SECTION}

2.1. Synthesis of MOF-5. All chemicals were purchased from Sigma-Aldrich and used without further purification. To synthesize MOF-5, we followed slightly modified scheme which was first reported by Yaghi et.al. ${ }^{12}$ Typically, $3.569 \mathrm{~g}$ of $\mathrm{Zn}\left(\mathrm{NO}_{3}\right)_{2} \cdot 6 \mathrm{H}_{2} \mathrm{O}(12 \mathrm{mmol})$ was dissolved in $40 \mathrm{~mL}$ of dimethylformamide (DMF) and stirred at room temperature for 15 minutes; meanwhile, in a separate bottle, $0.665 \mathrm{~g}$ of terephthalic acid $\left(\mathrm{H}_{2} \mathrm{BDC}\right)(4 \mathrm{mmol})$ and 3 $\mathrm{mL}$ of triethylamine (TEA) were dissolved in $60 \mathrm{~mL} \mathrm{DMF}$ and stirred for $15 \mathrm{~min}$ at room 
temperature. Then the solution of $\mathrm{Zn}\left(\mathrm{NO}_{3}\right)_{2} \cdot 6 \mathrm{H}_{2} \mathrm{O}$ was poured slowly into the $\mathrm{H}_{2} \mathrm{BDC}$ solution under constant stirring. The mixed solution was transferred into a $250 \mathrm{~mL}$ screw jar, which was closed tightly and put into an oil bath at $110^{\circ} \mathrm{C}$ for 20 hours under constant stirring. After cooling down, the white product was washed with DMF and chloroform twice and collected by centrifugation. The obtained products were dried in a vacuum oven at $80^{\circ} \mathrm{C}$ overnight.

2.2. MOF-5 Derived ZnO/C Nanocomposites. The as-prepared MOF-5 was used as precursor to prepare porous $\mathrm{ZnO} / \mathrm{C}$ nanocomposites. For this purpose, $0.20 \mathrm{~g}$ of MOF-5 was loaded in an alumina boat and placed in the center of a flow-through quartz tube sitting in a tube furnace. The furnace was set at a ramp rate of $10{ }^{\circ} \mathrm{C} / \mathrm{min}$ to the target temperature with a dwell time of 1 hour for each sample. The first series of four composites were obtained at $800^{\circ} \mathrm{C}$ in air, argon, argon saturated with water vapors introduced only during 1 hour dwell time (for short time duration; S) and argon with saturated water vapors introduced throughout the entire heat treatment (for longer time duration; L) and the resulting samples were named as $\mathrm{ZnO}-\mathrm{Air} 800$, $\mathrm{ZnO} / \mathrm{C}-\mathrm{Ar}_{800}, \mathrm{ZnO} / \mathrm{C}-\mathrm{Ar}+\mathrm{W}(\mathrm{S})_{800}$ and $\mathrm{ZnO} / \mathrm{C}-\mathrm{Ar}+\mathrm{W}(\mathrm{L})_{800}$ respectively. Following the same gaseous atmospheres and protocols, the second series of four composites were prepared at 1000 ${ }^{\circ} \mathrm{C}$ and the resulting samples were labelled as $\mathrm{ZnO}-\mathrm{Air}_{1000}, \mathrm{C}-\mathrm{Ar}_{1000}, \mathrm{ZnO} / \mathrm{C}-\mathrm{Ar}+\mathrm{W}(\mathrm{S})_{1000}$ and $\mathrm{ZnO} / \mathrm{C}-\mathrm{Ar}+\mathrm{W}(\mathrm{L})_{1000}$ respectively.

2.3. Material Characterization. The as-synthesized composites were used for characterizations and photocatalytic applications without further treatment. ${ }^{27-29}$ Powder X-ray diffraction (PXRD) patterns were recorded by employing Bruker D8 instrument with $\mathrm{Cu} \mathrm{K} \alpha$ radiation $(1.54 \AA)(40 \mathrm{kV}, 40 \mathrm{~mA})$. The powder samples were prepared by gently pressing the powder onto a glass slide. For MOF-5, XRD pattern were recorded in the $2 \theta$ range from $5^{\circ}$ to $40^{\circ}$ whereas the PXRD patterns of the MOF derived composites were measured in the $2 \theta$ range from $10^{\circ}$ to $80^{\circ}$. The morphologies, elemental compositions and elemental mapping of the asprepared composites were characterized by scanning electron microscopy (SEM) (Hitachi S3200N) and transmission electron microscopy (TEM) (JEM 2100 LeB6 $200 \mathrm{KV}$ ) equipped with energy dispersive X-ray spectroscopy (EDS). For SEM, powder samples were spread on a carbon tape and sputtered with a gold layer of $5 \mathrm{~nm}$ thickness. For TEM images and elemental mapping, the samples were first dispersed in absolute ethanol under moderate sonication and then pipetted onto a holey carbon $\mathrm{Cu}$ grid. Thermogravimetric analysis and mass spectroscopy 
(TGA-MS) were carried out on a TA SDT Q600 instrument coupled with Hiden QGA gas analysis mass spectrometer to detect the gas compositions from the exhaust emission. For TGAMS, $10 \mathrm{mg}$ of each sample was put into an alumina crucible and heated from 20 to $810{ }^{\circ} \mathrm{C}$ with a heating rate of $10^{\circ} \mathrm{C} / \mathrm{min}$ under an air flow of $100 \mathrm{~mL} / \mathrm{min}$. The Raman spectra were recorded (WITec ALPHA300 R) by using $532 \mathrm{~nm}$ laser excitation under $6 \mathrm{~mW}$ in a range from 20 to 2000 $\mathrm{cm}^{-1}$. Fourier-transform infrared (FTIR) spectra of the samples were recorded with an Alpha Bruker system. For each sample, a small amount of sample was mixed with $\mathrm{KBr}$ powder and pressed in the form of a pallet for the FTIR analysis. The X-ray photoelectron spectroscopy (XPS) was performed using a Kratos AXIS ULTRA spectrometer with a monochromated Al KR X-ray source $(1486.6 \mathrm{eV})$ operated at $10 \mathrm{~mA}$ emission current and $15 \mathrm{kV}$ anode potential. The analysis chamber pressure was better than $1.3 \times 10^{-12}$ bar. The take-off angle for the photoelectron analyzer was $90^{\circ}$ and the acceptance angle was $30^{\circ}$. The energy band gaps of the $\mathrm{ZnO}$ and $\mathrm{ZnO} / \mathrm{C}$ nanocomposites were calculated from diffuse reflectance spectra acquired by employing a spectrophotometer (PerkinElmer lambda 1050 with $150 \mathrm{~mm}$ integrated InGaAs sphere). The specific surface areas (SSA) and the pore size distributions (PSD) of the composites were measured on a Micromeritics 3Flex instrument. Prior to the measurements the samples were degassed at $200{ }^{\circ} \mathrm{C}$ for 3 hours. The $\mathrm{N}_{2}$ gas used had a purity of $99.999 \%$. The BET surface area was calculated using the Rouquerol correction for microporous solids. The pore size distribution was determined using nonlocal DFT with the Tarazona model for nanoporous materials with cylindrical pores.

2.4. Evaluation of Adsorption/Photodegradation Activity. Adsorption of methylene blue (MB) from water solution on the as-synthesized $\mathrm{ZnO} / \mathrm{C}$ nanocomposites was evaluated in a dark box. Typically, $10 \mathrm{mg}$ of as-prepared sample was added into $50 \mathrm{~mL}$ of $20 \mathrm{ppm}$ aqueous $\mathrm{MB}$ solution. The suspension was immediately transferred to a dark box under constant stirring. After different intervals of time, $1 \mathrm{~mL}$ aliquots were taken and the concentration of $\mathrm{MB}$ in the solution was determined by UV-vis absorption spectroscopy. Using a similar procedure, the synergistic adsorption and photodegradation of $\mathrm{MB}$ by $\mathrm{ZnO} / \mathrm{C}$ composites were measured under visible light irradiation. The visible light source (cutoff filter, $\lambda>420 \mathrm{~nm}$ ) was provided by 10 lamps of $20 \mathrm{~W}$ with a light intensity of $160 \mathrm{~mW} / \mathrm{cm}^{2}$ (PerfectLight, $320 \mathrm{~nm} \leq \lambda \leq 780 \mathrm{~nm}$ ). The adsorption/photodegradation of MB (\%) was calculated by using the following expression: 
$\mathrm{D}(\%)=\frac{\mathrm{C}_{\mathrm{o}}-\mathrm{C}}{\mathrm{C}_{\mathrm{o}}} \times 100 \%$

Where $\mathrm{D}$ is the percentage of degradation, $\mathrm{C}_{\mathrm{o}}$ is the initial concentration and $\mathrm{C}$ is the final concentration. ${ }^{29}$ To measure the recyclability and stability of the material, the best performing sample $\mathbf{Z n O / C - A r + W ( S )})_{1000}$ was separated from solution by filtration using a filter paper. Before reusing for the next run, the filtered sample was washed with deionized water several times and dried in oven at $110{ }^{\circ} \mathrm{C}$ for 2 hours. The sample was consecutively used for 5 cycles.

\section{RESULTS AND DISCUSSION}

3.1. Characterizations of the Composites. The crystal structures and phase transformations of the nanocrystalline MOF-5 and obtained derivatives were examined by Powder X-ray diffraction (PXRD) as shown in Figure 1. The PXRD pattern of as-synthesized MOF-5 (Figure S1) exhibits reflections at $2 \theta=6.8^{\circ}$ and $9.7^{\circ}$, which is in agreement with the theoretically predicted PXRD pattern of MOF-5 synthesized by the direct mixing method. ${ }^{28-31}$

Heat processing of MOF-5 at high temperature in air flow results in white colored pure $\mathrm{ZnO}$ nanoparticles. The samples ZnO-Airsoo and ZnO-Air1000 show diffraction peaks at $2 \theta$ of $31.7^{\circ}, 34.4^{\circ}, 36.2^{\circ}, 47.5^{\circ}, 56.5^{\circ}, 62.8^{\circ}, 66.3^{\circ}, 67.9^{\circ}, 72.5^{\circ}$ and $76.9^{\circ}$ which confirm the polycrystalline wurtzite structure of $\mathrm{ZnO}^{27,}{ }^{32} \mathrm{ZnO}$-Air ${ }_{1000}$ shows much stronger diffraction peaks than ZnO-Air800, indicating higher crystallinity of the $\mathrm{ZnO}$ nanoparticles. However, black products were generated upon heat treatment in argon and water vapors, signifying the carbonization of precursor. Heat processing of MOF-5 in Ar atmosphere, a significant amount of $\mathrm{ZnO}$ appears in the sample $\mathbf{Z n O} / \mathbf{C}$-Ar800 whereas no $\mathrm{ZnO}$ is observed in the PXRD patterns of sample C-Ar 1000. This may be due to the fact that while the heat process temperature is above the boiling point of $\mathrm{Zn}\left(907^{\circ} \mathrm{C}\right)$, the formed $\mathrm{Zn}$ is likely to evaporate. Consequently, no diffraction peaks belonging to $\mathrm{Zn}$ or $\mathrm{ZnO}$ species can be observed in the powder diffraction pattern of $\mathbf{C}$ Ar1000. A broad reflection centered at around $2 \theta=23^{\circ}$ is due to the (002) lattice plane reflection of graphitic carbon species. The broadening of this diffraction peak indicates the low degree of graphitization, confirming the amorphous nature of the formed carbon. ${ }^{30-33}$ It can be observed that the diffraction peak intensities of $\mathrm{ZnO} / \mathrm{C}$ are weaker as compared to pure $\mathrm{ZnO}$ samples. It indicates that the samples obtained in $\mathrm{Ar}$ and $\mathrm{H}_{2} \mathrm{O}$ atmosphere at $800{ }^{\circ} \mathrm{C}$ possess relatively lower 
crystallinity and smaller particle sizes. The closer observation (Inset to Figure 1a) shows that in sample $\mathbf{Z n O / C - A r + W ( S ) 8 0 0}$ the strongest diffraction peak (101) of $\mathrm{ZnO}$ shifts slightly from $36.24^{\circ}$ to $36.18^{\circ} 2 \theta$. Such diffraction shifting compared to pure $\mathrm{ZnO}$ is assigned to doping induced unit cell deformation. It is further confirmed by DRS, FTIR and Raman spectroscopy that change in $\mathrm{ZnO}$ lattice parameters and structural deformation can be attributed to the induced defects and narrow energy band gap.

The nanocomposite $\mathbf{Z n O / C - A r + W ( L ) 1 0 0 0}$ shows broad reflections representative for amorphous carbon as well as the very low intensity peaks of $\mathrm{ZnO}$. However, the $\mathbf{Z n O} / \mathbf{C}$ Ar+W(S)1000 showed only the broader diffraction peak of amorphous carbon. The intensities of the $\mathrm{ZnO}$ reflections in all samples obtained in $\mathrm{Ar}$ and $\mathrm{H}_{2} \mathrm{O}$ atmosphere at $1000{ }^{\circ} \mathrm{C}$ are negligible compared with the samples prepared at $800{ }^{\circ} \mathrm{C}$, most likely due to the relative very low content of $\mathrm{ZnO}$ in those samples, as shown in the TGA-MS measurements below. Compared with sample C-Ar1000, the diffraction peaks of amorphous carbon for the water vapors processed samples remained unaffected by water vapors atmosphere that clearly indicated the preservation of a carbon structures under these conditions, which was also confirmed by Raman spectroscopy and XPS analysis. Due to the high intensity of the $\mathrm{ZnO}$ reflections for all the composites derived at $800{ }^{\circ} \mathrm{C}$, the diffraction peak for amorphous carbon is suppressed. From the PXRD results, it is evident that the heat process temperature as well as the heat treatment atmosphere during the carbonization process has a significant impact on the nature of the resulting metal oxide/carbon nanocomposites.
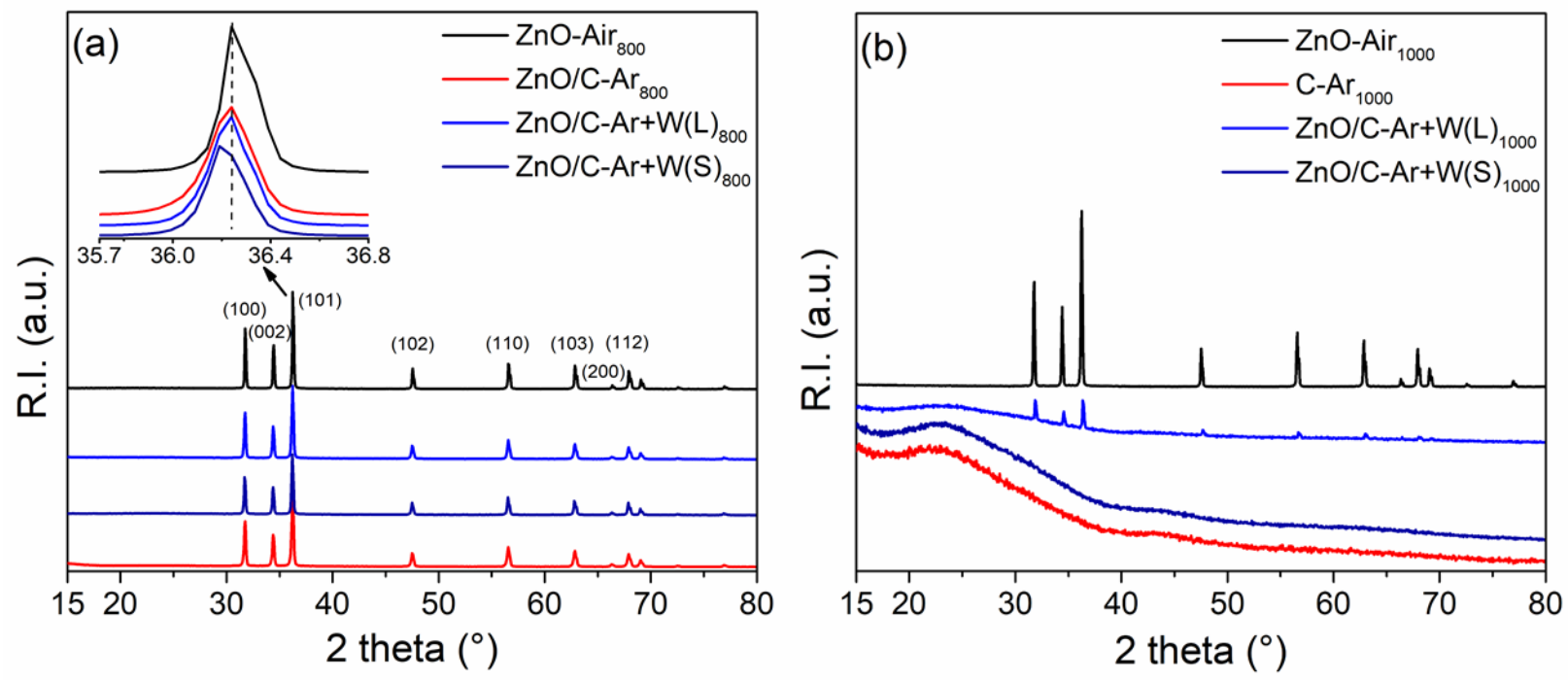
Figure 1. PXRD patterns of nanocomposites obtained in different carbonization atmospheres at $800{ }^{\circ} \mathrm{C}$ (a) and $1000{ }^{\circ} \mathrm{C}$ (b). ZnO-Air, ZnO/C-Ar+W(L), ZnO/C-Ar+W(S) and ZnO/C-Ar are represented in black, light blue, dark blue and red, respectively. Numbers in brackets mark the miller indices of the lattice plane reflections of the wurtzite modification of $\mathrm{ZnO}$. Inset to Figure 1(a) is the amplified PXRD pattern of peak (101).

TGA-MS was carried out under air flow to evaluate the thermal stabilities of nanocomposites as well as to determine the amount of carbon and other species presented in the as-synthesized nanocomposites. The TGA profile of the as-synthesized MOF-5 in Figure 2a shows $20 \%$ weight loss in the first step at around $200{ }^{\circ} \mathrm{C}$ due to the evaporation of the solvent $\mathrm{DMF}$ and adsorbed moisture and further $40 \%$ weight loss in the second step at around $500{ }^{\circ} \mathrm{C}$ due to the decomposition of the organic linker.
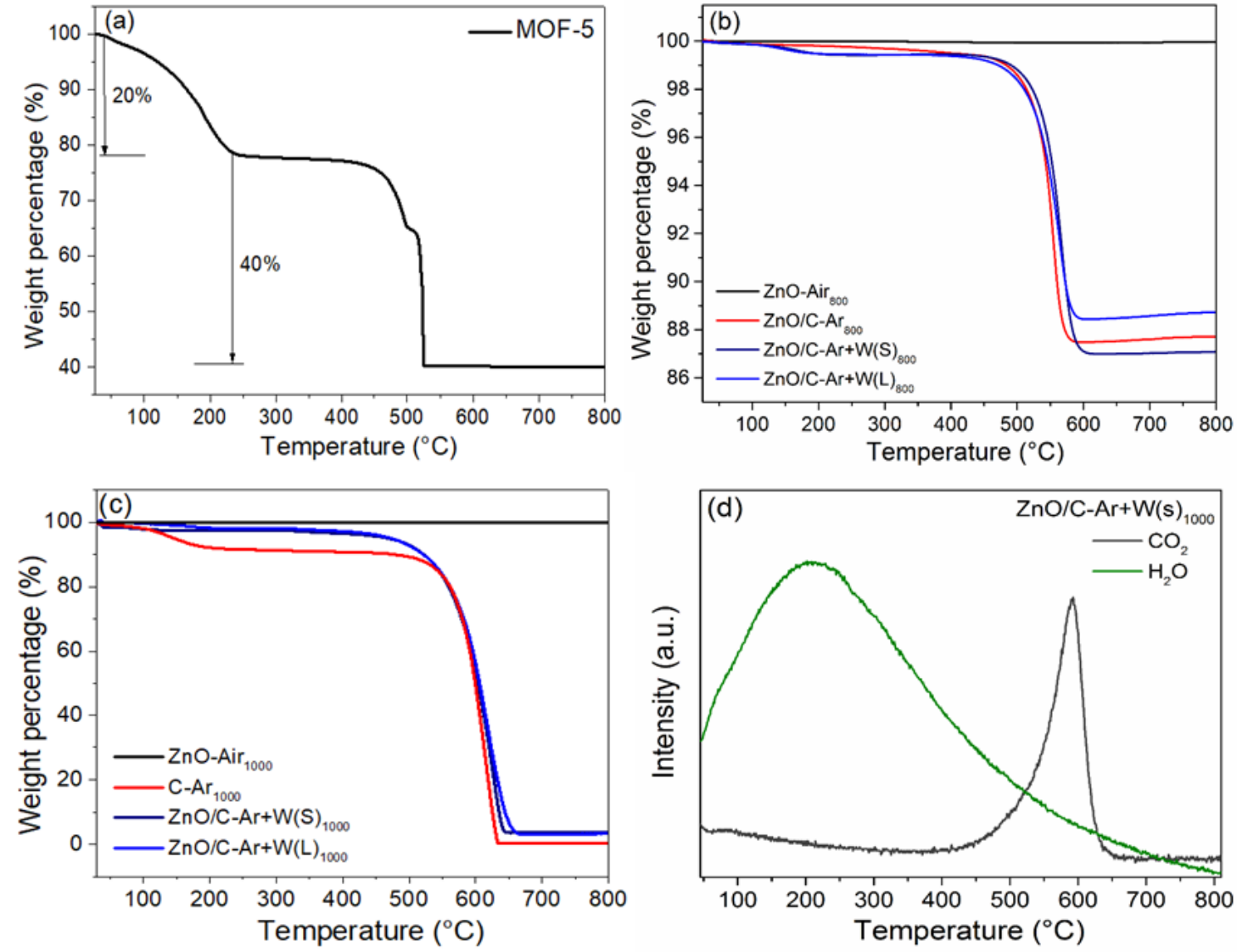

Figure 2. TGA profiles of MOF-5 (a) and derived composites (ZnO-Air (black), ZnO/C$\mathbf{A r}+\mathbf{W}(\mathbf{L})$ (blue), $\mathbf{Z n O} / \mathbf{C}-\mathbf{A r}+\mathbf{W}(\mathbf{S})$ (dark blue) and $\mathbf{Z n O} / \mathbf{C}-\mathbf{A r}$ (red)) obtained under different 
gaseous atmospheres at $800{ }^{\circ} \mathrm{C}(\mathrm{b}), 1000{ }^{\circ} \mathrm{C}$ (c) and temperature dependent mass spectra (d) surveying $\mathrm{H}_{2} \mathrm{O}$ and $\mathrm{CO}_{2}$ release during heat treatment of $\mathbf{Z n O} / \mathbf{C}-\mathbf{A r}+\mathbf{W}(\mathbf{s})_{1000}$.

Figure $2 \mathrm{~b}$ and $\mathrm{c}$ represent the TGA profiles of composites obtained at 800 and $1000{ }^{\circ} \mathrm{C}$ respectively. ZnO-Air800 and ZnO-Air1000 are pure wurtzite polycrystalline $\mathrm{ZnO}$ derived from MOF-5 and show no weight loss in the applied temperature range, confirming the absence of carbon in these samples. However, sample C-Ar1000 lost around $10 \%$ mass at $100{ }^{\circ} \mathrm{C}$, implying the removal of adsorbed moisture and further weight loss of the remaining $90 \%$ mass at around $650{ }^{\circ} \mathrm{C}$, indicating that only carbon is presented in this sample with no residual species. This is clearly due to the complete evaporation of $\mathrm{Zn}$ species during the carbonization process that results in the generation of pure porous carbon. The composites $\mathbf{Z n O / C - A r 8 0 0 , ~ Z n O / C - ~}$ $\mathbf{A r}+\mathbf{W}(\mathbf{S}) 800$ and $\mathbf{Z n O} / \mathbf{C}-\mathbf{A r}+\mathbf{W}(\mathbf{L}) \mathbf{8 0 0}$ exhibit $11 \%$ to $13 \%$ weight loss at around $550{ }^{\circ} \mathrm{C}$ due to the burn-off of carbon, which suggests that these composites contain up to $87-89 \%$ of $\mathrm{ZnO}$. However, the composites $\mathbf{Z n O} / \mathbf{C}-\mathbf{A r}+\mathbf{W}(\mathbf{S}) \mathbf{1 0 0 0}$ and $\mathbf{Z n O} / \mathbf{C}-\mathbf{A r}+\mathbf{W}(\mathbf{L}) \mathbf{1 0 0 0}$ contain around 96\% of carbon. The mass spectra in Figure 2(d) further confirms that the weight loss upon heating in air is due to the release of adsorbed moisture $\left(\mathrm{H}_{2} \mathrm{O}\right)$ at lower temperature and burn-off of carbon to form $\mathrm{CO}_{2}$ at higher temperature up to $600{ }^{\circ} \mathrm{C}$. Due to the presence of oxygen species in the carbonization atmosphere, a very samll amount of $\mathrm{ZnO}$ was retained in the composites regardless of the carbonization temperatures. The obtained TGA-MS results show good consistency with the PXRD patterns. It is observed that the short time water exposure (S) and longer time moisture exposure during the entire carbonization process $(\mathbf{L})$ has less influence on the $\mathrm{ZnO}$ content in the composites. However, the longer exposure to moisture has considerable impact on the specific surface areas and functionalization of composites as confirmed by BET, FTIR and Raman spectroscopies. The composites derived at $1000{ }^{\circ} \mathrm{C}$ demonstrated increased thermal stability in comparison to the composites obtained at $800{ }^{\circ} \mathrm{C}$.

The morphologies of the MOF-5 precursor and its derived composites were analyzed by scanning electron microscopy (SEM) and transmission electron microscopy (TEM). Welldefined cubic crystals of MOF-5, approximately $1 \mu \mathrm{m}$ in size are clearly observed in the SEM micrographs shown in Figure $3 \mathrm{a}$ and $\mathrm{b}$. Upon heating to elevated temperatures and carbonization, the material retained its cubic morphology which is in agreement with previous reports. ${ }^{34}$ In spite of different carbonization atmospheres, no morphological differences are observed in samples 
synthesized at $800{ }^{\circ} \mathrm{C}$. However, at treatment temperatures of $1000{ }^{\circ} \mathrm{C}$, most of the $\mathrm{ZnO}$ reduced to $\mathrm{Zn}$ and evaporated, leaving behind smaller porous carbon particles with high surface areas confirmed by gas sorption analysis. More hollow and partially broken cubic porous carbon structures can be seen in Figure 3d. Srinivas et al. argued that this process can be called a selfactivation of carbon, which is predominantly influenced by the framework structures, metal centers and initial porosities of the precursor MOFs. ${ }^{34}$ The SEM images of samples ZnO/CArsov and $\mathbf{Z n O} / \mathbf{C}-\mathbf{A r}+\mathbf{W}(\mathbf{L}) \mathbf{8 0 0}$ are shown in Figure S5a and b confirming that the morphologies of the composites are retained in all atmospheric conditions. After using of composite $\mathbf{Z n O} / \mathbf{C}$ $\operatorname{Ar}+\mathbf{W}(\mathbf{S})_{800}$ for dye photodegradation, SEM images were taken (Figure S5c and d). No visible morphological changes are observed.
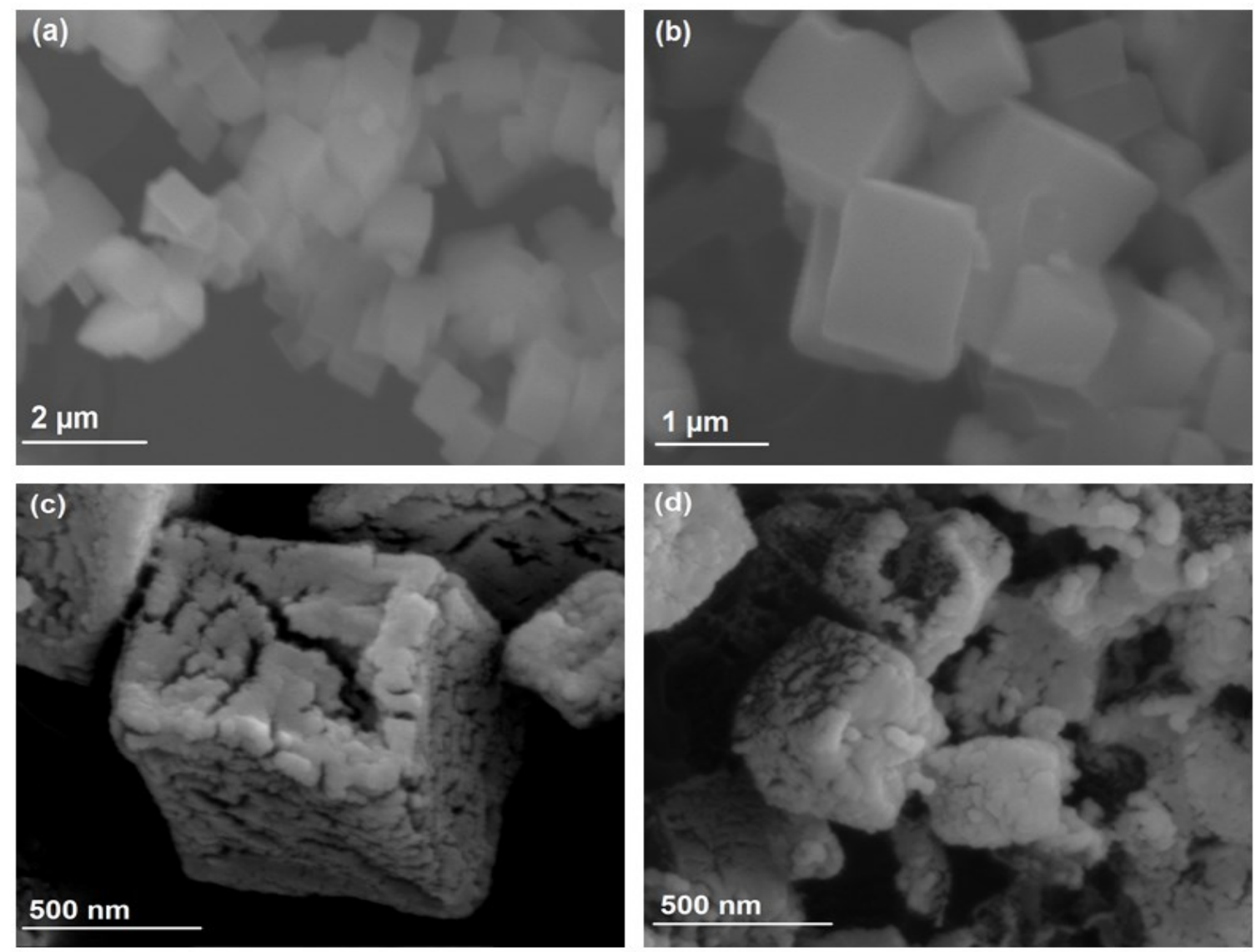

Figure 3. Representative SEM micrographs of $\operatorname{MOF}-5$ (a, b), $\mathbf{Z n O} / \mathbf{C}-\mathbf{A r}+\mathbf{W}(\mathbf{S}) 800$ (c) and ZnO/C-Ar+W(S)1000 (d). 
Representative TEM images of selected composites $\mathbf{Z n O / C - A r + W ( S ) 8 0 0 ~ a n d ~} \mathbf{Z n O / C -}$ $\mathbf{A r}+\mathbf{W}(\mathbf{S})_{1000}$ are presented in Figure 4. The low magnification images (Figure 4a and d confirm the topology of the derived composites. In the High-resolution TEM (HR-TEM) images (Figure $4 \mathrm{c}$ and $\mathrm{f}$, the nanosized $\mathrm{ZnO}$ particles embedded in the transparent layers of porous carbon are evident. The estimated sizes of these $\mathrm{ZnO}$ nanoparticles lie in the range of 5-10 nm. Figure $\mathrm{S} 6$ shows the HRTEM image of selected sample $\mathbf{Z n O / C - A r + W ( s ) 8 0 0 ~ w h e r e ~ t h e ~ f r i n g e s ~ o f ~} \mathrm{ZnO}$ crystal lattice can be clearly seen. Figure 5.The homogeneous dispersion of $\mathrm{ZnO}$ nanoparticles (Figure 5) in porous carbon matrices is further confirmed by elemental mapping. The composite $\mathbf{Z n O} / \mathbf{C}-\mathbf{A r}+\mathbf{W}(\mathbf{S})_{800}$ exhibits homogeneous distribution of zinc, oxygen and carbon throughout the selected sample area. The elemental mapping of the composite $\mathbf{Z n O / C - A r + W ( S )})_{1000}$ shows the uniform dispersion of $\mathrm{C}$ with negligible amount of $\mathrm{Zn}$ and $\mathrm{O}$, due to the high $\mathrm{C}$ content of 96\% in this sample. As confirmed by XRD and TGA (Figure 1a and Figure 2b), homogeneously distributed $\mathrm{ZnO}$ nanoparticles in a porous carbon framework can be achieved upon hightemperature carbonization of highly crystalline MOF- 5 in water saturated argon atmosphere. ${ }^{27}$ The nanoparticles with such small sizes usually show broader XRD peaks. However, in these samples, the peaks are of relatively high intensity. It can be understood in this way that we use MOF-5 as precursor, which contains $\mathrm{Zn}, \mathrm{C}, \mathrm{O}$ and during the carbonization process, the benzene rings will carbonize to form amorphous porous carbon, while the $\mathrm{Zn}$ will migrate to combine with $\mathrm{O}$ to form $\mathrm{ZnO}$. Although many of the formed $\mathrm{ZnO}$ particles with small size (such as 5-10 $\mathrm{nm}$ ) are surrounded by the formed amorphous porous carbon, under high temperature pyrolysis conditions, some small $\mathrm{ZnO}$ particles are inevitably migrate together to form bigger $\mathrm{ZnO}$ particles due to the high surface energy of the small $\mathrm{ZnO}$ particles. Therefore in the $\mathrm{XRD}$, the strong diffraction peaks from the bigger $\mathrm{ZnO}$ makes it difficult to see the contribution from small particles. In Figure $4 \mathrm{~b}$ and e, the dark spots of isolated bigger $\mathrm{ZnO}$ nanoparticles can be seen. 

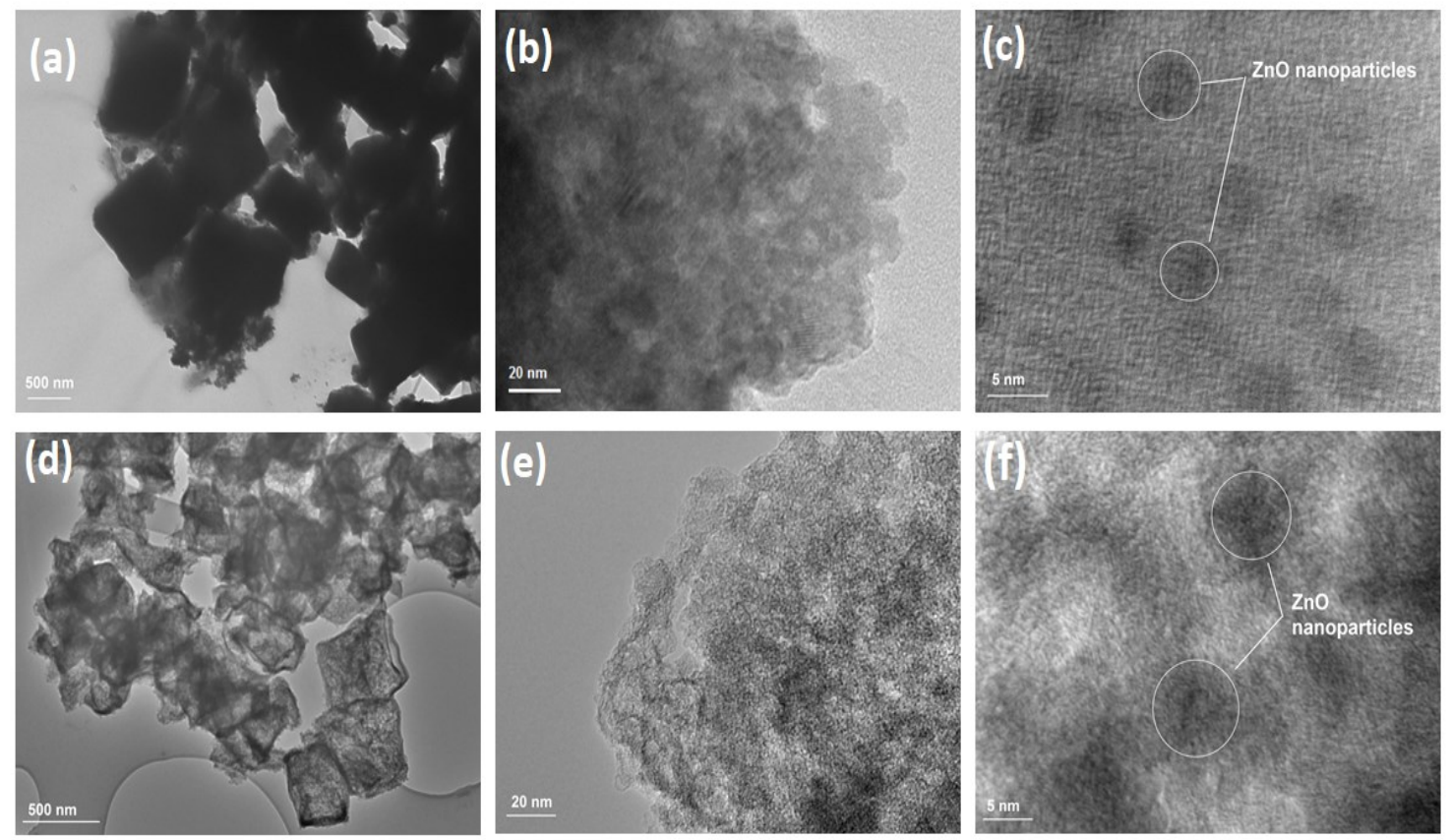

Figure 4. TEM and HRTEM images of $\mathbf{Z n O} / \mathbf{C}-\mathbf{A r}+\mathbf{W}(\mathbf{S}) \mathbf{8 0 0}(\mathrm{a}, \mathrm{b}, \mathrm{c})$ and $\mathbf{Z n O} / \mathbf{C}-\mathbf{A r}+\mathbf{W}(\mathbf{S}) \mathbf{1 0 0 0}$ $(\mathrm{d}, \mathrm{e}, \mathrm{f})$ at different magnifications.
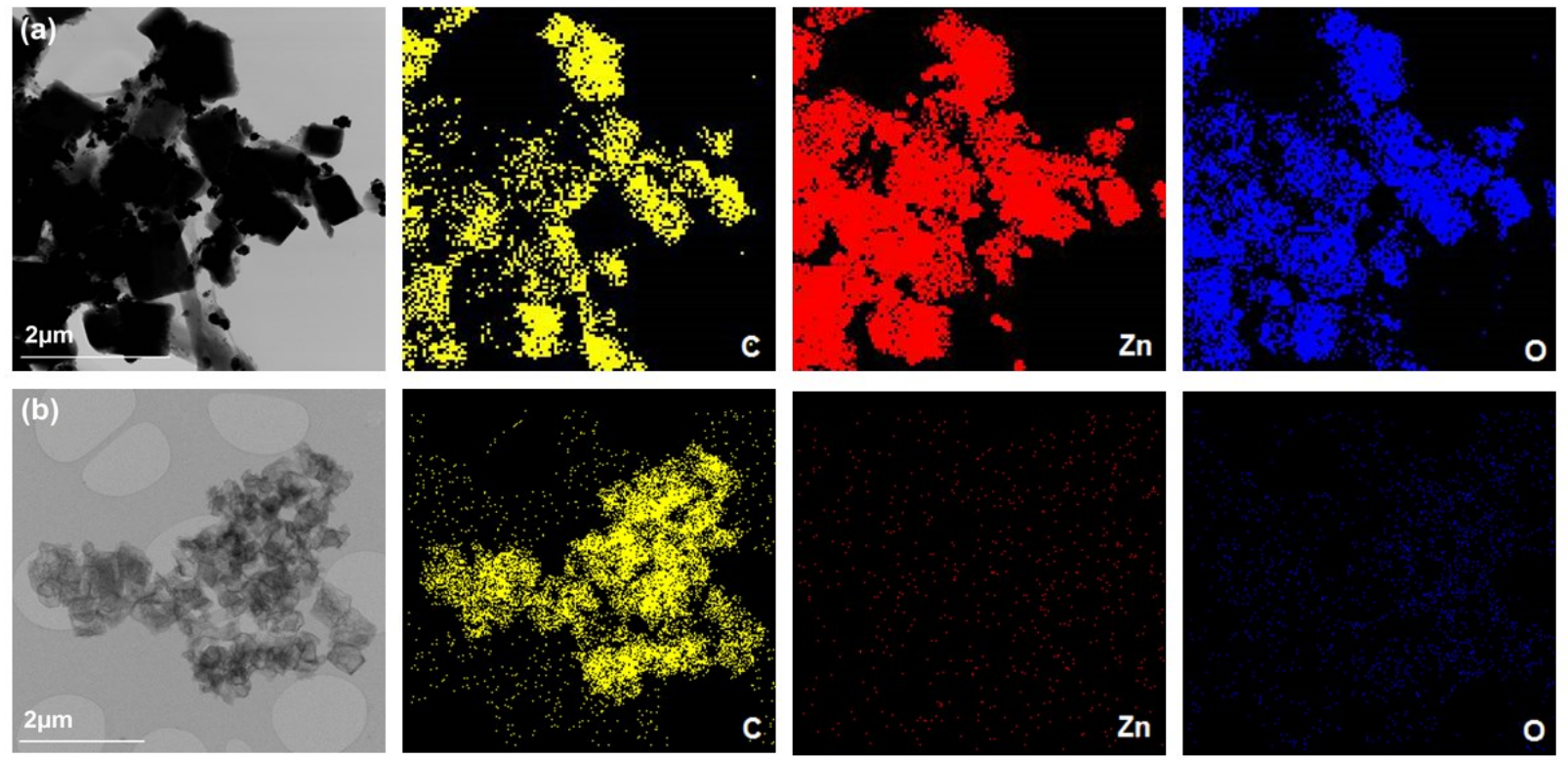

Figure 5. TEM micrographs and elemental maps of $\mathbf{Z n O} / \mathbf{C}-\mathbf{A r}+\mathbf{W}(\mathbf{S}) \mathbf{8 0 0}$ (a) and $\mathbf{Z n O} / \mathbf{C}$ Ar+W(S)1000 (b). The elemental maps were obtained by EDS. The colored images show the 
distribution of elements throughout the composite. $\mathrm{C}, \mathrm{Zn}$, and $\mathrm{O}$ are represented in yellow, red and blue, respectively.

To study the nature of the carbon presented in these nanocomposites and lattice vibrational modes of $\mathrm{ZnO}$, Raman spectra of all materials were recorded (Figure 6a and Figure S2a). The composites ZnO-Air800 and ZnO-Air1000 show strong signals for highly crystalline MOF-5 derived pure $\mathrm{ZnO}$ nanoparticles with main peaks occurring at 438 and $454 \mathrm{~cm}^{-1}$ due to $\mathrm{E}_{2}$ (high) mode respectively. The other peaks at around 332 and $347 \mathrm{~cm}^{-1}$ in these samples correspond to $\mathrm{A}_{1}$ (TO) mode (second order Raman processes) of $\mathrm{ZnO}$. For bulk $\mathrm{ZnO}$, these signals appear at $475 \mathrm{~cm}^{-1} .{ }^{35-38}$ The $\mathrm{E}_{2}$ phonon frequency usually occurs at around $436 \mathrm{~cm}^{-1}$ for ZnO quantum dots (QDs) with sizes less than $10 \mathrm{~nm} \cdot{ }^{37}$ The observed $\mathrm{ZnO}$ peaks indirectly confirms the particle sizes observed by HRTEM. It should be noted that in MOF-5 derived $\mathrm{ZnO} / \mathrm{C}$ at $800{ }^{\circ} \mathrm{C}$, these $\mathrm{E}_{2}$ (high) and $\mathrm{A}_{1}(\mathrm{TO})$ modes red shifted to 427 and $321 \mathrm{~cm}^{-1}$ compared to pure $\mathrm{ZnO}$ (Inset to Figure 6a). The observed red shift (to lower wavenumbers) in mode positions can be ascribed to the strain on the $\mathrm{ZnO}$ due to the presence of carbon atoms in the crystal lattice. Red shift in Raman spectra also takes place when the incident photons lose energy upon interaction with the vibrational modes of the material (phonons). This energy is absorbed by the material leading to the peak shift toward lower frequencies. It suggests that the doped carbon atoms alter the bond lengths and the lattice parameters of $\mathrm{ZnO}$, generating new crystalline lattice defects and eventually narrowing the energy band gap. This red shift in frequencies is also observed in FTIR spectra of $\mathrm{ZnO} / \mathrm{C}$ composites shown in Figure $\mathrm{S} 3$.

Two characteristic signals of amorphous carbon are observed at around 1350 and $1590 \mathrm{~cm}^{-}$ ${ }^{1}$ for all samples obtained under argon and water vapor atmosphere at 800 and $1000{ }^{\circ} \mathrm{C}$. In the samples ZnO/C-Ar800, ZnO/C-Ar+W(S)800 and ZnO/C-Ar+W(L)800, the G band around 1588 $\mathrm{cm}^{-1}$ is generated by the formation of nanocrystalline carbon, and represents the bond stretching of all $\mathrm{sp}^{2}$ atoms in both, all carbon hexagonal rings and chains. The D band at around $1348 \mathrm{~cm}^{-1}$ is due to the breathing modes of $\mathrm{sp}^{2}$ hybridized carbon atoms in hexagonal rings, which signifies the formation of amorphous carbon. This mode is forbidden in perfect graphite crystals and it can only be seen in the presence of disorder in structure. The intensity of the D band and particularly the $\mathrm{I}_{\mathrm{D}} / \mathrm{I}_{\mathrm{G}}$ ratio is a measure for the content of defects and disorder in the crystalline carbon structures. ${ }^{39-41}$ 
As shown in Figure 6a and Figure $\mathrm{S} 2 \mathrm{a}$, the comparison of the $\mathrm{I}_{\mathrm{D}} / \mathrm{I}_{\mathrm{G}}$ ratios of the composites ZnO/C-Arsoo (0.82), ZnO/C-Ar+W(S)800 (0.82) and ZnO/C-Ar+W(L)800 (0.86) with C-Ar1000 (0.99), $\mathbf{Z n O / C - A r + W ( S ) 1 0 0 0 ~ ( 0 . 8 9 ) ~ a n d ~} \mathbf{Z n O / C - A r + W ( L ) 1 0 0 0 ~ ( 0 . 8 4 ) ~ r e v e a l ~ t h a t ~ i n c r e a s i n g ~ t h e ~}$ annealing temperature increases the $\mathrm{I}_{\mathrm{D}} / \mathrm{I}_{\mathrm{G}}$ ratio which indicates that the high temperature and longer exposure to the water vapors during the carbonization process generate more defects in the carbon matrix, eventually enhancing the amorphous nature of the composites. ${ }^{41-43}$
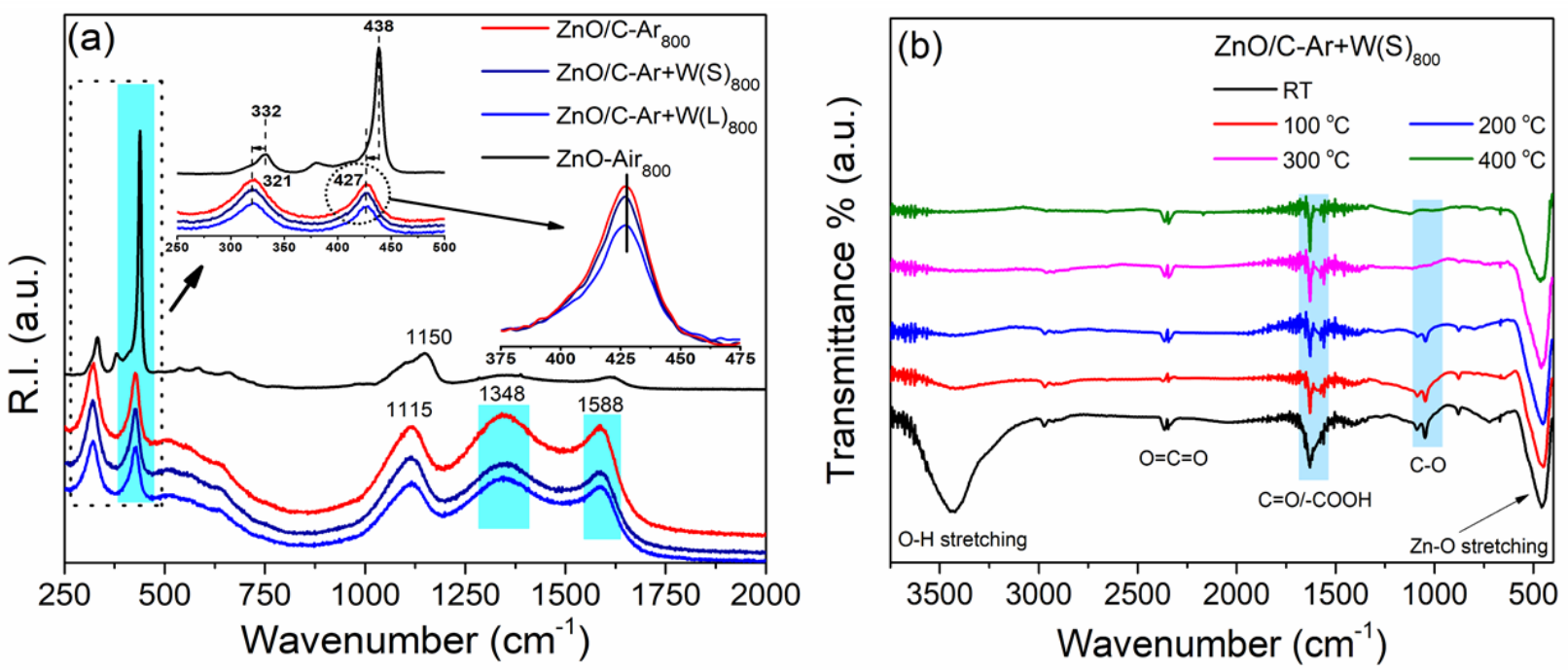

Figure 6. Raman Spectra of ZnO-Air (black), ZnO/C-Ar (red), ZnO/C-Ar+W(S) (dark blue) and $\mathbf{Z n O} / \mathbf{C}-\mathbf{A r}+\mathbf{W}(\mathbf{L})$ (light blue) prepared at $800^{\circ} \mathrm{C}$ (a). FTIR spectra of $\mathbf{Z n O} / \mathbf{C}-\mathbf{A r}+\mathbf{W}(\mathbf{S})$ derived at $800{ }^{\circ} \mathrm{C}$ (b) after treatment of the materials at RT (black), $100{ }^{\circ} \mathrm{C}$ (red), $200{ }^{\circ} \mathrm{C}$ (blue), $300{ }^{\circ} \mathrm{C}$ (pink) and $400{ }^{\circ} \mathrm{C}$ (green), respectively.

FTIR was employed to investigate the presence of functional groups on the surface of the nanocomposites. The FTIR spectra of selected samples $\mathbf{Z n O} / \mathbf{C}-\mathbf{A r}+\mathbf{W}(\mathbf{s}) \mathbf{8 0 0}$ and $\mathbf{Z n O} / \mathbf{C}-$ $\mathbf{A r}+\mathbf{W}(\mathbf{s}) 1000$ were recorded at room temperature. As shown in Figure $6 \mathrm{~b}, \mathbf{Z n O} / \mathbf{C}-\mathbf{A r}+\mathbf{W}(\mathbf{s}) 800$ exhibits a strong band at $460 \mathrm{~cm}^{-1}$ which is attributed to the $\mathrm{Zn}-\mathrm{O}$ stretching from the crystalline wurtzite $\mathrm{ZnO}$. This signal in $\mathbf{Z n O} / \mathbf{C}-\mathbf{A r}+\mathbf{W}(\mathbf{s}) \mathbf{1 0 0 0}$ is negligible (shown in Figure $\mathrm{S} 2 \mathrm{~b}$ ), due to the much lower $\mathrm{ZnO}$ content in this sample, as demonstrated in XRD, XPS and TGA analysis. A strong signal is observed at around $1630 \mathrm{~cm}^{-1}$ in both samples, which is assigned to the $\mathrm{C}-\mathrm{OH}$ and $\mathrm{C}=\mathrm{O}$ bond stretching of $-\mathrm{OH}$ and $-\mathrm{COOH}$ groups. A comparatively weak band at $2360 \mathrm{~cm}^{-1}$ in these samples is ascribed to $\mathrm{O}=\mathrm{C}=\mathrm{O}$ bond stretching. A broader signal at $3445 \mathrm{~cm}^{-1}$ indicative for $\mathrm{O}-\mathrm{H} / \mathrm{Zn}-\mathrm{OH}$ bond stretching of adsorbed $\mathrm{H}_{2} \mathrm{O}$ is observed in both samples. ${ }^{1}$ 
To confirm the presence of carboxylate $(-\mathrm{COOH})$ groups on the surface of the nanocomposites, the sample was heated in a furnace at $100,200,300$ and $400{ }^{\circ} \mathrm{C}$ for 1 hour and then the FTIR spectra were recorded subsequently. It was found that the signal at $1630 \mathrm{~cm}^{-1}$ became more prominent in both samples during heating, which confirms the presence of - $\mathrm{COOH}$ functional groups. This effect is accompanied by the decrease of the broad $\mathrm{O}-\mathrm{H}$ stretching vibrations at $3445 \mathrm{~cm}^{-1}$ and $\mathrm{C}-\mathrm{H}$ stretching at $2972 \mathrm{~cm}^{-1}$, indicating the gradual evaporation of the adsorbed $\mathrm{H}_{2} \mathrm{O}$. The FTIR spectra of ZnO-Ar800, ZnO-Ar+W(S)800, ZnO/C-Ar+W(L)800 and ZnO-Air800 are presented in Figure S3. The relatively stronger peak at $1630 \mathrm{~cm}^{-1}$ in $\mathbf{Z n O}$ $\operatorname{Ar}+\mathbf{W}(\mathbf{S}) 800$ (Inset to Figure S3) confirms that there is higher concentration of $-\mathrm{COOH}$ functional group present in this sample compared to other prepared composites.

To further analyze the elemental compositions of the derived composites, the XPS spectra of selected samples are presented. As shown in Figure 7a, the wide elemental spectra confirm the presence of $\mathrm{Zn}, \mathrm{O}$ and $\mathrm{C}$ in all the composites obtained at $800^{\circ} \mathrm{C}$. In the $\mathrm{Zn} 2 \mathrm{p}$ spectrum, the $\mathrm{Zn}$ $2 p_{3 / 2}$ and $2 p_{1 / 2}$ doublet (Figure $7 b$ ) appear at binding energies of 1021.05 and $1044.15 \mathrm{eV}$, respectively. The binding energy distance between $2 p_{3 / 2}$ and $2 p_{1 / 2}(23.1 \mathrm{eV})$ energy levels implies the presence of $\mathrm{Zn}^{2+}$ ions. The $\mathrm{Zn}$ peak is stronger in pure $\mathrm{ZnO}$ sample whereas the intensity reduces in composites $\mathbf{Z n O / C - A r 8 0 0}$ and $\mathbf{Z n O / C - A r + W ( S ) 8 0 0 ~ w i t h ~ a ~ b l u e ~ s h i f t ~ i n ~ b i n d i n g ~ e n e r g y ~}$ from 1021.05 to $1021.15 \mathrm{eV}$, maybe due to the carbon doping into the $\mathrm{ZnO}$ lattice.

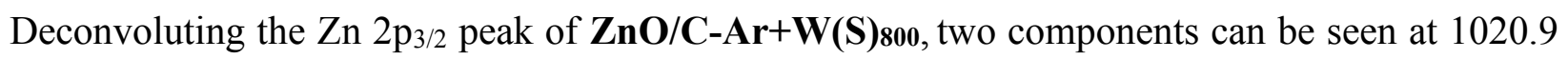
and $1021.2 \mathrm{eV}$ which can be ascribed to $\mathrm{Zn}-\mathrm{O}-\mathrm{C}$ due to the doped carbon and $\mathrm{Zn}-\mathrm{O}$ bond respectively. ${ }^{44}$ As shown in Figure 7c, the main peak of $\mathrm{O}_{1 \mathrm{~s}}$ in sample ZnO-Airsoo is observed at $530.13 \mathrm{eV}$ with a small shoulder at $531.59 \mathrm{eV}$. These peaks are allocated to the $\mathrm{Zn}-\mathrm{O}$ bond and surface adsorbed oxygen species, respectively. ${ }^{7}$ For the sample $\mathbf{Z n O / C - A r 8 0 0 , ~ O 1 s ~ p e a k ~ a t ~}$ 530.35 and $531.74 \mathrm{eV}$ can be ascribed to $\mathrm{Zn}-\mathrm{O}$ bond, metal carbonate species such as $\mathrm{C}-\mathrm{O} / \mathrm{C}=\mathrm{O}$ bonds or surface adsorbed oxygen species respectively. However, for the sample ZnO/C$\mathbf{A r}+\mathbf{W}(\mathbf{S})_{800}, \mathrm{O}_{1 \mathrm{~s}}$ peaks can be deconvoluted into three separate peaks centered at 530.27, 530.99 and $532.19 \mathrm{eV}$. These peaks can be assigned to the $\mathrm{Zn}-\mathrm{O}$ bond, carbonate species $(\mathrm{C}-\mathrm{O}) / \mathrm{C}=\mathrm{O})$ and carboxylic functional group $-\mathrm{COOH}$ introduced through water steam atmosphere during carbonization of MOF-5 respectively. ${ }^{45,46}$ 

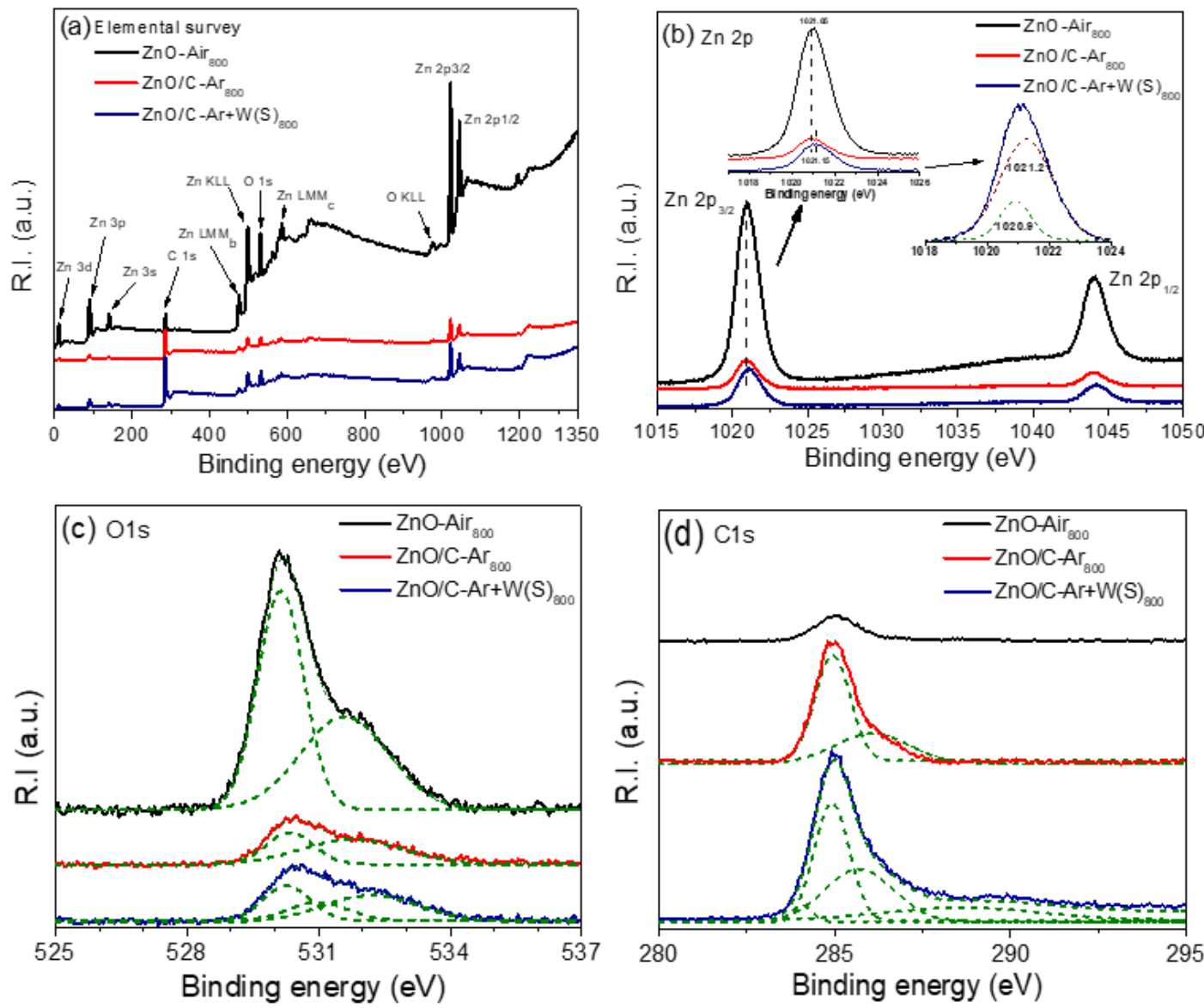

Figure 7. XPS spectra of elemental survey (a) Zn 2p (b) O 1s (c) C 1s (d) for ZnO-Air800 (black) and ZnO/C-Arsoo (red) and ZnO/C-Ar+W(S)800(Dark blue).

The $\mathrm{C}_{1 \mathrm{~s}}$ spectra (Figure 7d) of samples $\mathbf{Z n O} / \mathbf{C}-\mathbf{A r} 800$ and $\mathbf{Z n O} / \mathbf{C}-\mathbf{A r}+\mathbf{W}(\mathbf{S})$ son show main peaks at $284.9 \mathrm{eV}$. It is in good agreement with the reported C-C bond of $\mathrm{sp}^{2}$ hybridized graphitic carbon confirmed by Raman results. ${ }^{47}$ The other deconvoluted peaks of C1s in ZnO/CArsoo and $\mathbf{Z n O} / \mathbf{C}-\mathbf{A r}+\mathbf{W}(\mathbf{S})_{800}$ appear at around $286 \mathrm{eV}$ can be ascribed to carbonate species with different amount of $\mathrm{C}=\mathrm{O} / \mathrm{C}-\mathrm{O}$ bonds. A small $\mathrm{C} 1 \mathrm{~s}$ peak appeared at $284.1 \mathrm{eV}$ in $\mathbf{Z n O} / \mathbf{C}$ $\mathbf{A r}+\mathbf{W}(\mathbf{S}) 800$ can be assigned to $\mathrm{Zn}-\mathrm{C}$ bond. ${ }^{48}$ The low intensity of this peak shows that there is only small amount of carbon is doped into the $\mathrm{ZnO}$ lattice. A broader peak at 288.9 arises due to the presence of $-\mathrm{COOH}$ functional group as confirmed by O1s XPS peak as well as FTIR spectra (Figure 6b). ${ }^{7}$ The higher intensity of $\mathrm{O}_{1 \mathrm{~s}}$ and $\mathrm{C} 1 \mathrm{~s}$ in $\mathbf{Z n O} / \mathbf{C}-\mathbf{A r}+\mathbf{W}(\mathbf{S}) \mathbf{8 0 0}$ as compared to ZnO/C-Ar800 provides the evidence of successful incorporation of other oxygen species (- 
$\mathrm{COOH}$ functional groups) into the composites. A low intensity peak for carbon in the pure $\mathbf{Z n O}$ Air800 is also observed at $285.1 \mathrm{eV}$, which may due to the background and sample holder.

The specific surface areas and pore size distributions of the nanocomposites were determined by $\mathrm{N}_{2}$ physisorption at $77 \mathrm{~K}$ and the isotherms are shown in Figure 8 and Figure S4 respectively. Due to the capillary filling of the micropores, all samples showed type I isotherms. As shown in Figure 8a and $\mathrm{b}$, the adsorption is reversible which verifies the predominant microporous nature of these composites. The composites $\mathbf{Z n O} / \mathbf{C}-\mathbf{A r} \mathbf{8 0 0}, \mathbf{Z n O} / \mathbf{C}-\mathbf{A r}+\mathbf{W}(\mathbf{S})_{800}$ and ZnO/C-Ar+W(L)800 exhibits BET surface areas of 369, 350 and $210 \mathrm{~m}^{2} / \mathrm{g}$ respectively. The BET surface areas of the composites C-Ar 1000, ZnO/C-Ar+W(S)1000 and ZnO/C-Ar+W(L)1000 derived at $1000^{\circ} \mathrm{C}$ are measured to be 2839,2390 and $2600 \mathrm{~m}^{2} / \mathrm{g}$ respectively, much higher than those samples derived from $800{ }^{\circ} \mathrm{C}$. These exceptional BET surface areas of composites $\mathbf{C}$ Ar1000, ZnO/C-Ar+W(S)1000 and $\mathbf{Z n O / C - A r + W ( L ) 1 0 0 0 ~ o r i g i n a t e ~ f r o m ~ t h e ~ v e r y ~ h i g h ~ s u r f a c e ~ a r e a ~}$ of the precursor MOF-5 $\left(3800 \mathrm{~m}^{2} / \mathrm{g}\right){ }^{22}$ Upon carbonization of MOF-5 in Ar above the boiling point of $\mathrm{Zn}$, the $\mathrm{Zn}$ metal atoms ionize and evaporate leaving behind highly porous amorphous carbon with partially broken inherited cubic morphology. The retained high porosity and defects in these derived 3D amorphous cubic carbons show high surface areas confirmed by SEM and HRTEM. However, the measured values are still lower than the reported precursor MOF-5.

It is worth noting that the specific surface areas of the composites obtained in Ar atmosphere are higher than the composites obtained in water vapors. It may be due to the water steam causing some $\mathrm{ZnO}$ to stay in the pores reducing the overall surface areas. TGA measurements confirmed that there is no $\mathrm{ZnO}$ nanoparticles in sample $\mathbf{C}$-Ar1000, therefore it has the highest specific surface area. In contrast, the nanocomposite $\mathbf{Z n O / C - A r + W ( L ) 8 0 0 ~ s h o w e d ~ t h e ~}$ lowest surface area among these samples. 

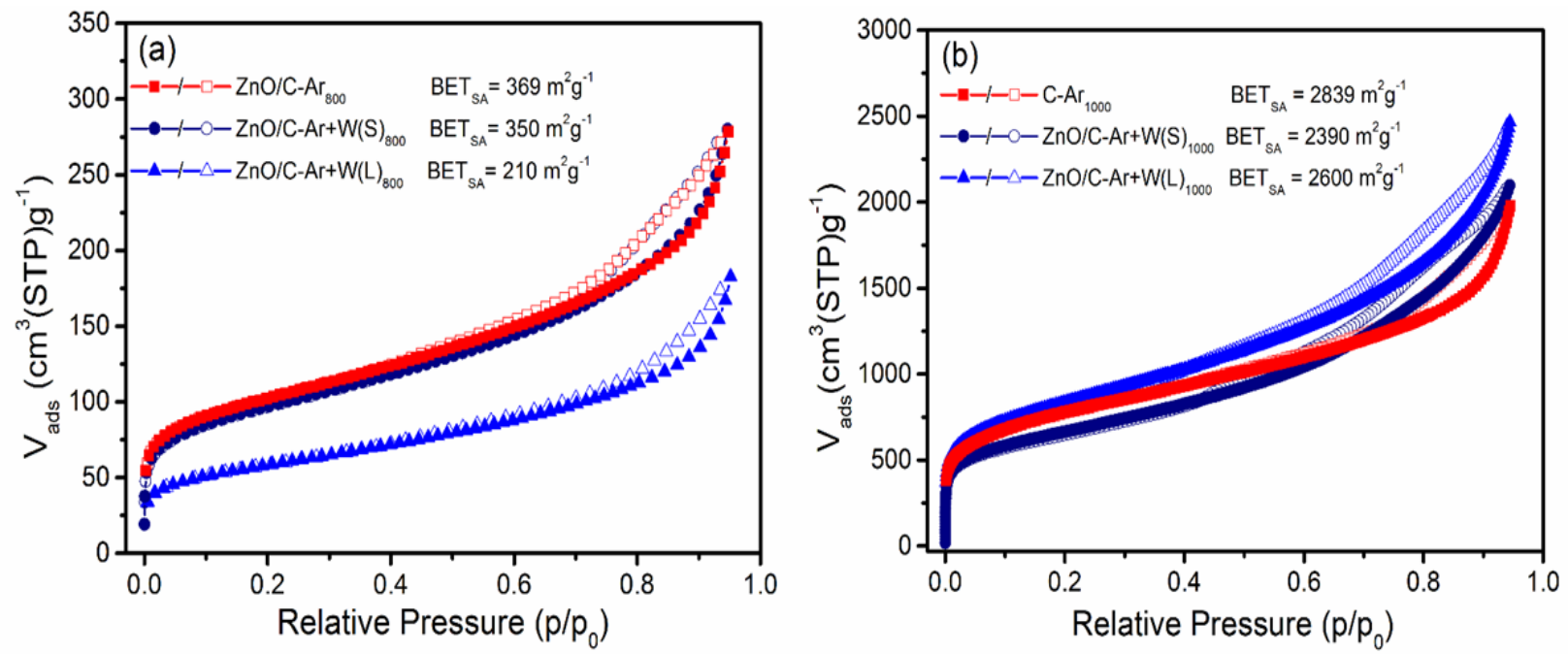

Figure 8. $\mathrm{N}_{2}$ sorption isotherms measured at $77 \mathrm{~K}$ for $\mathbf{Z n O} / \mathbf{C}$-Ar and $\mathbf{C}$-Ar (red squares), $\mathbf{Z n O} / \mathbf{C}-\mathbf{A r}+\mathbf{W}(\mathbf{S})$ (dark blue circles) and $\mathbf{Z n O} / \mathbf{C}-\mathbf{A r}+\mathbf{W}(\mathbf{L})$ (light blue triangles) derived at 800 ${ }^{\circ} \mathrm{C}$ (a) and $1000{ }^{\circ} \mathrm{C}$ (b) respectively. Filled and empty symbols represent adsorption and desorption. The lines are a guide to the eye only.

Pore size distributions (PSD) of these nanocomposites were analyzed based on the nitrogen adsorption data using the Non Local Density Functional Theory (NLDFT) model. As shown in Figure S4a and $\mathrm{b}$, the nanocomposites derived at $800^{\circ} \mathrm{C}$ and $1000^{\circ} \mathrm{C}$ are dominated with PSDs between 10 and $15 \AA$ centering at around $12.3 \AA$. It also confirms that all the derived composites are predominantly microporous regardless of the atmosphere applied.

3.2. Optical Properties and Photocatalytic Dye Degradation Performance. The optical properties of the composites were studied by employing UV-vis diffuse reflectance spectroscopy (DRS). The reflectance spectra of selected nanocomposites are shown in Figure 9a. The reflectance spectra of the composites $\mathbf{Z n O} / \mathbf{C}-\mathbf{A r}+\mathbf{W}(\mathbf{S}) \mathbf{8 0 0}$ and $\mathbf{Z n O} / \mathbf{C}-\mathbf{A r}+\mathbf{W}(\mathbf{S}) \mathbf{1 0 0 0}$ red shifts to 387 and $395 \mathrm{~nm}$ respectively in comparison to pristine ZnO-Air1000 which exhibits the reflectance at $380 \mathrm{~nm}$. The energy band gaps (EBG) of the composites were calculated by using the Tauc plot as shown in Figure 9b. The EBG of MOF-5 derived ZnO-Air 1000 was determined as $3.26 \mathrm{eV}$ which is smaller than the $\mathrm{EBG}$ of commercially available $\mathrm{ZnO}$ nanoparticles (3.37 $\mathrm{eV}$ ). The EBG of $\mathbf{Z n O} / \mathbf{C}-\mathbf{A r}+\mathbf{W}(\mathbf{S})_{\mathbf{8 0 0}}$ and $\mathbf{Z n O} / \mathbf{C}-\mathbf{A r}+\mathbf{W}(\mathbf{S})_{1000}$ were calculated as $3.0 \mathrm{eV}$ and $3.1 \mathrm{eV}$ respectively. The MOF-5 derived nanocomposites $\mathbf{Z n O} / \mathbf{C}-\mathbf{A r}+\mathbf{W}(\mathbf{S}) \mathbf{8 0 0}$ and $\mathbf{Z n O} / \mathbf{C}-$ Ar+W(S)1000 show considerable shifts in EBGs compared to the EBG of the bulk ZnO. The 
proposed mechanism of dye photodegradation by $\mathrm{ZnO} / \mathrm{C}$ composites is shown in Figure 10. Due to the doping of anionic carbon, the valance band of $\mathrm{ZnO}$ shifts upwards, resulting the narrow EBG. It should be noted that the EBG strongly correlates with the size and morphology of the nanoparticles. MOF-5 derived $\mathrm{ZnO}$ nanoparticles retain the cubic particle shapes of MOF-5 with sizes in the range of 5-10 $\mathrm{nm}$. Therefore, the EBG was predictable to be shifted to longer wavelengths confirmed by Raman, FTIR and XPS results. It can be expected that with the decrease of energy band gaps, these homogeneously distributed $\mathrm{ZnO}$ nanoparticles in porous carbon can absorb more visible light which will result in an improved photocatalytic performance.
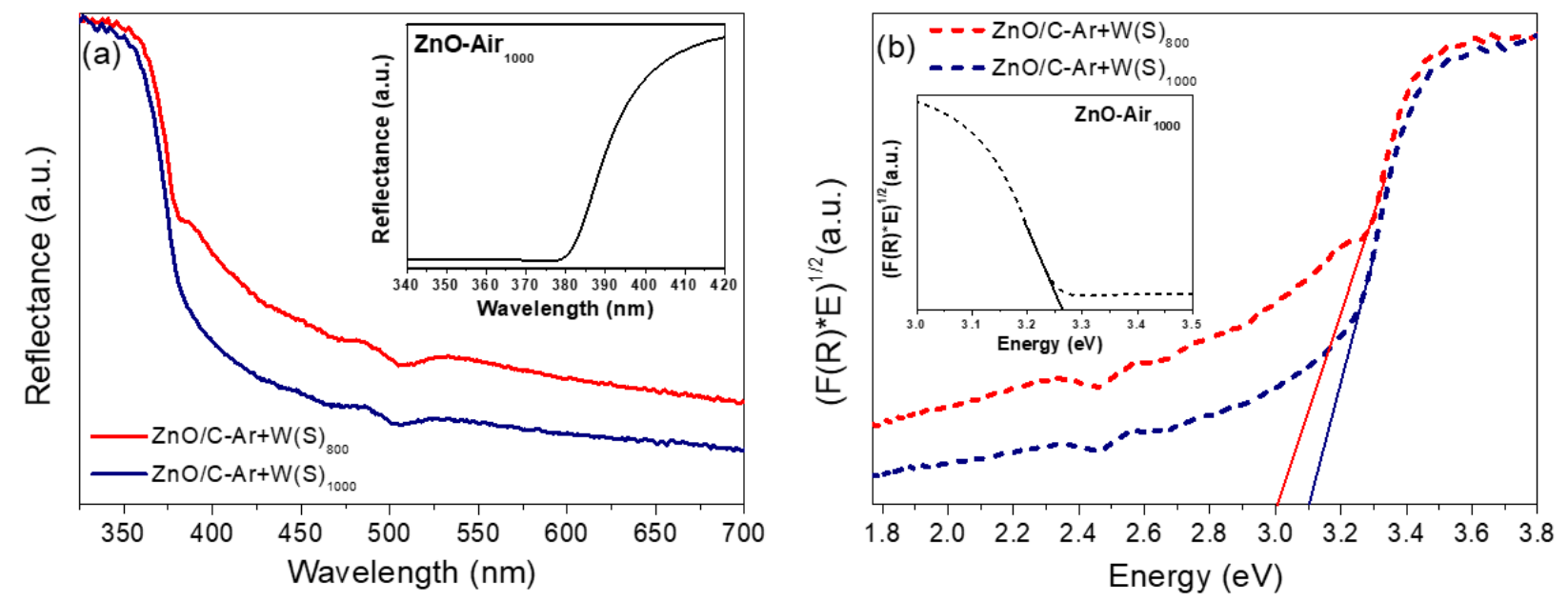

Figure 9: Reflectance spectra of selected samples ZnO-Airirooo (black, insets), ZnO/C$\mathbf{A r}+\mathbf{W}(\mathbf{S}) 800$ (red) and $\mathbf{Z n O} / \mathbf{C}-\mathbf{A r}+\mathbf{W}(\mathbf{S})_{1000}$ (dark blue) (a) and energy band gaps of these composites calculated by the Kubelka-Munk function (Tauc plots) (b). 


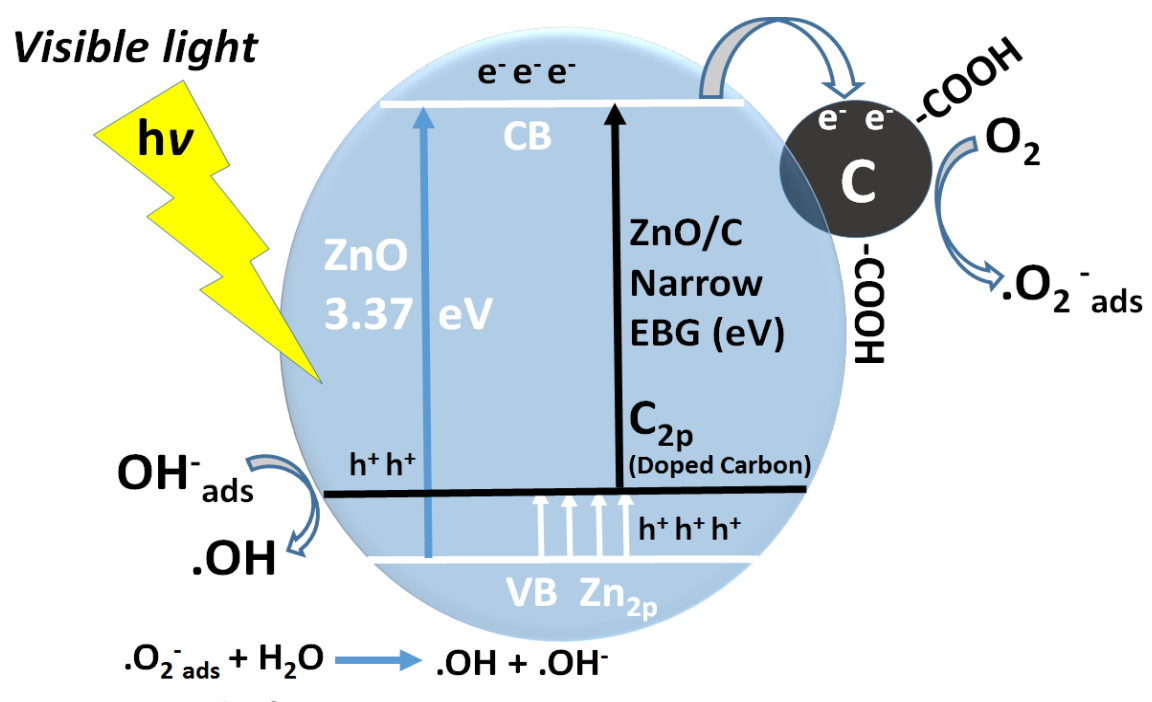

$. \mathrm{OH}+$ Organic dye $\longrightarrow$ Photodegradation products $+\mathrm{CO}_{2}+\mathrm{H}_{2} \mathrm{O}$

Figure 10: Mechanism of energy band gap shift in MOF derived $\mathrm{ZnO} / \mathrm{C}$ nanocomposites and the dye photodegradation under visible light irradiation.

To demonstrate the adsorption and photodegradation capacities of the as-synthesized porous $\mathrm{ZnO} / \mathrm{C}$ nanocomposites, the degradation of methylene blue (MB) was carried out in a dark room and under visible light irradiation respectively. To evaluate the photocatalytic activity of each sample, $10 \mathrm{mg}$ of as-prepared sample was added to $50 \mathrm{ml}$ of $20 \mathrm{ppm} \mathrm{MB}$ aqueous solution and the mixture was stirred for 3 hours. As shown in Figure 11a under dark conditions, catalysts $\mathbf{Z n O} / \mathbf{C}-\mathbf{A r 8 0 0}, \mathbf{Z n O} / \mathbf{C}-\mathbf{A r}+\mathbf{W}(\mathbf{S}) 800$ and $\mathbf{Z n O} / \mathbf{C}-\mathbf{A r}+\mathbf{W}(\mathbf{L}) \mathbf{8 0 0}$ demonstrate substantial amount of dye adsorption up to 28, 53 and $34 \%$ respectively. On the contrary, ZnO-Airso0 exhibits almost no adsorption. The samples C-Ar1000, ZnO/C-Ar+W(S)1000 and ZnO/CAr+W(L)1000 (Figure 11c) adsorb 79, 89 and $86 \%$ of the dye under dark room conditions respectively, but ZnO-Air1000 shows no adsorption. Moreover, most of MB was adsorbed on the surface of the porous composites in the first 30 to 60 minutes before achieving an adsorption/desorption equilibrium. Obviously, the observed high degradation of MB in dark room conditions is mainly due to the high textural properties of the as-synthesized $\mathrm{ZnO} / \mathrm{C}$ composites, which is consistent with our previous report. ${ }^{27}$ To measure the photocatalytic performance of the composites, the photodegradation of MB was carried out under visible light irradiation. While sample ZnO-Air800 displayed 25\% of MB photodegradation in 3 hours, samples ZnO/C-Ar800, ZnO/C-Ar+W(S)800 and ZnO/C-Ar+W(L)800 showed adsorption and 
photodegradation of 82, 99 and $92 \%$, respectively (Figure 11b). Compared with sample ZnOAir800, sample ZnO-Air1000 only showed $7.5 \%$ photodegradation capacity under visible light irradiation, maybe due to the formation of large $\mathrm{ZnO}$ particles at higher heat process temperature. However, Figure 11d shows that the composites C-Ar1000, ZnO/C-Ar+W(S)1000 and ZnO/CAr+W(L)1000 exhibited 80, 99 and $85 \%$ of adsorption and photocatalytic degeneration of MB respectively. Comparing the photocatalytic activity of best performing composites $\mathbf{Z n O / C}$ $\mathbf{A r}+\mathbf{W}(\mathbf{S}) 800$ and $\mathbf{Z n O} / \mathbf{C}-\mathbf{A r}+\mathbf{W}(\mathbf{S})_{1000}$ under dark and visible light, it can be clearly observed that the photodegradation of MB is more dominant in $\mathbf{Z n O} / \mathbf{C}-\mathbf{A r}+\mathbf{W}(\mathbf{S}) \mathbf{8 0 0}$ due to high content of $\mathrm{ZnO}$ whereas adsorption is more dominant in $\mathbf{Z n O} / \mathbf{C}-\mathbf{A r}+\mathbf{W}(\mathbf{S})_{1000}$ due to high content of carbon. In UV-vis absorption spectra, it is difficult to differentiate between simultaneous adsorption and photodegradation. It is evident that under the most suitable synthesis conditions the composites $\mathbf{Z n O} / \mathbf{C}-\mathbf{A r}+\mathbf{W}(\mathbf{S}) \mathbf{8 0 0}$ and $\mathbf{Z n O} / \mathbf{C}-\mathbf{A r}+\mathbf{W}(\mathbf{S}) \mathbf{1 0 0 0}$ with homogeneously dispersed and carbon doped $\mathrm{ZnO}$ nanoparticles in the highly porous amorphous carbon framework show the best adsorption and photodegradation performance. Apparently, high surface area composite should show highest adsorption. However, these samples have not demonstrated best adsorption and dye degradation of MB. Confirmed by various characterization techniques (i.e. XRD, HRTEM, Raman spectroscopy, XPS and FTIR) not only surface areas but other parameters also play crucial role in adsorption and dye photodegradation. The presence of carboxylate $(-\mathrm{COOH})$ functional groups and oxygen species on the surface of the catalysts provides active sites that increase the hydrophilic behavior and facilitates the MB molecules to be accommodated inside the pores and subsequently degraded, which consequently result in higher photocatalytic performance of composites derived from water vapor atmosphere than the ones prepared in argon atmosphere. It can be inferred that the porous framework, the hydrophilic functional groups on the surface of porous carbons, carbon doping and the homogeneous dispersion of $\mathrm{ZnO}$ nanoparticles play a significant role for the enhancement of adsorption and photocatalytic performance for dye degradation. 

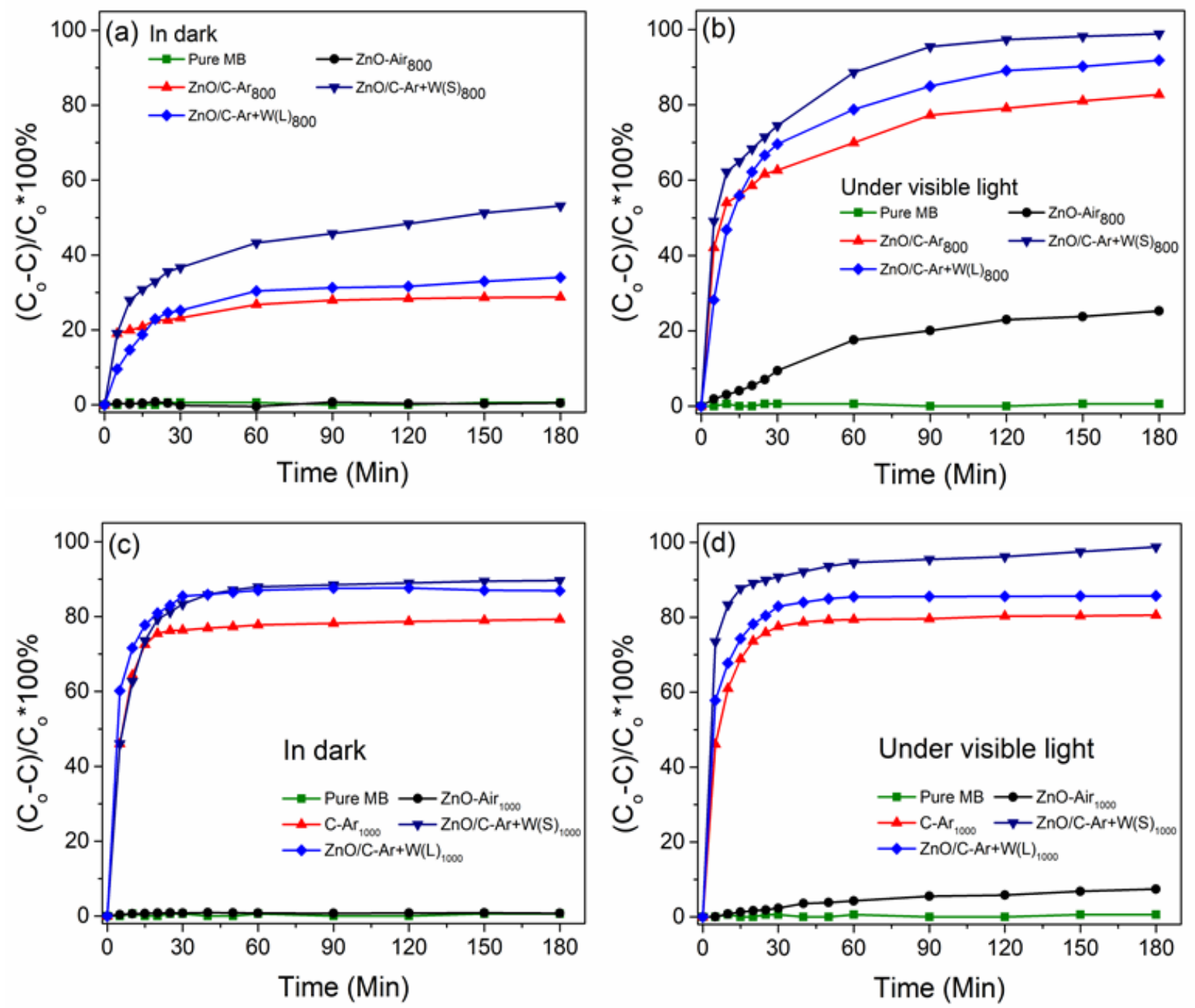

Figure 11. The adsorption and photodegradation of $\mathrm{MB}$ by ZnO-Air (black circles), ZnO/CAr(red triangles), $\mathbf{Z n O} / \mathbf{C}-\mathbf{A r}+\mathbf{W}(\mathbf{L})$ (light blue diamonds) and $\mathbf{Z n O} / \mathbf{C}-\mathbf{A r}+\mathbf{W}(\mathbf{S})$ (dark blue triangles) derived at $800^{\circ} \mathrm{C}$ and $1000^{\circ} \mathrm{C}$ in dark (a, c) and under visible light irradiation (b, d) respectively. Green squares represent blind test without any catalysts.

It has been reported that the decolorization of organic dyes could take place due to a selfsensitization mechanism. ${ }^{1}$ Organic dyes show light absorption in visible light region, therefore the charge excitation takes place in the organic dye molecules, resulting in the formation of unstable cation radicals on the surface of the $\mathrm{ZnO}$ semiconductor. These generated active species attack the dye molecules leading to the decolorization of the dye without mineralization. The newly produced secondary products could be toxic. This mechanism can prevent the completely 
mineralization of the organic dye into neutral species such as $\mathrm{CO}_{2}$ and $\mathrm{H}_{2} \mathrm{O}$ by actual photocatalysis. ${ }^{1}$

To confirm that the MB was completely neutralized by photocatalytic degradation, $25 \mathrm{~mL}$ of pure $\mathrm{MB}$ was placed under the visible light for 3 hours and the intensity of the absorption peaks was recorded by UV-vis spectrophotometry. There was no change in intensity of the MB for 3 hours which verified that the MB did not self-sensitize as shown in Figure 11a-d.

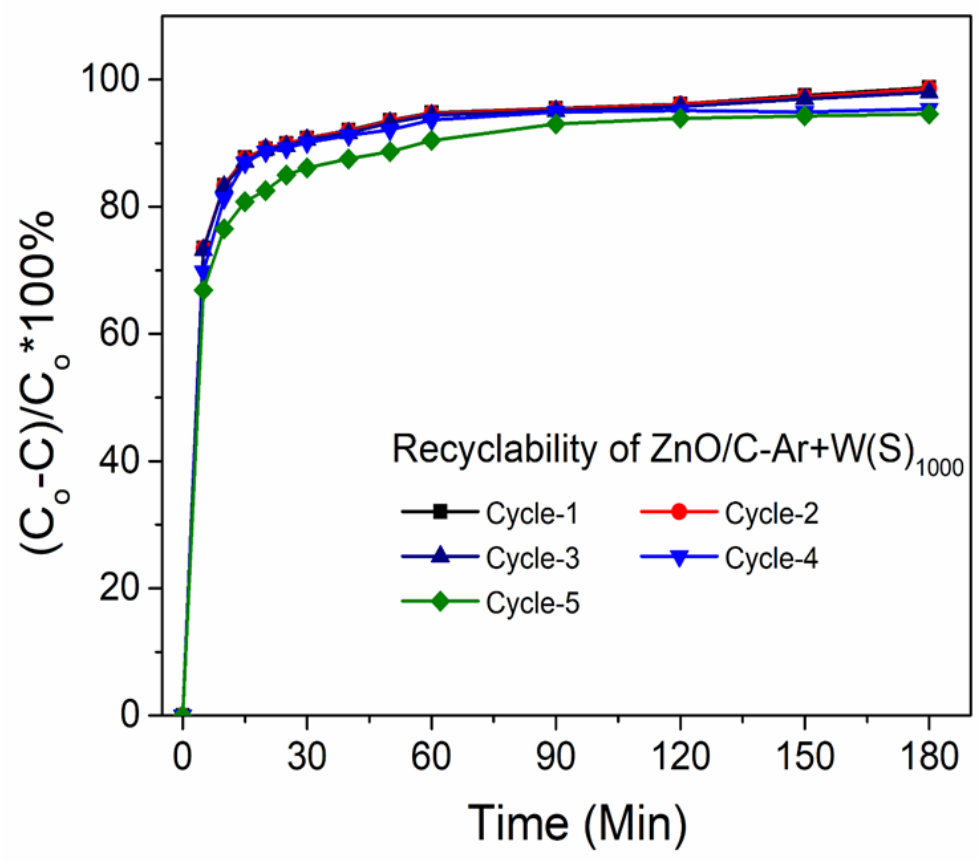

Figure 12. Recyclability test of the composite $\mathbf{Z n O} / \mathbf{C}-\mathbf{A r}+\mathbf{W}(\mathbf{S}) \mathbf{1 0 0 0}$ for the adsorption and photodegradation of MB in water under visible-light irradiation.

To study the stability and reusability of the composites, $\mathbf{Z n O} / \mathbf{C}-\mathbf{A r}+\mathbf{W}(\mathbf{S}) \mathbf{1 0 0 0}$ was tested in 5 consecutive MB degradation cycles. For this reusability test, $30 \mathrm{mg}$ of the catalyst was put into $150 \mathrm{~mL}$ of $20 \mathrm{ppm}$ MB solution and stirred for 3 hours. After each cycle, the used catalyst was filtered from the solution, washed several times with deionized water and dried over night at 110 ${ }^{\circ} \mathrm{C}$ before using for the next run. Figure 12 shows that the nanocomposite retains the efficiency over the whole five cycles with only negligible decreases. The $5^{\text {th }}$ cycle showed still $94 \%$ photodegradation, signifying a good stability and reusability of the $\mathbf{Z n O} / \mathbf{C}-\mathbf{A r}+\mathbf{W}(\mathbf{S}) 1000$ nanocomposite. It can be established that the MOF derived $\mathrm{ZnO} / \mathrm{C}$ nanocomposites do not form 
bonds with water molecules and the microporous structure of composite remains intact, preventing the agglomeration of $\mathrm{ZnO}$ nanoparticles. In addition, the UV-vis absorption spectra were shown in Figure S7 confirmed that the absorption intensity decreases with time during the photocatalytic process. The MB absorbance remains at the same wavelength which indicates that no secondary products were formed due to the decolorization of the dye molecules. Eventually MB dye molecules are completely mineralized and led to the completely removal of MB from water, accompanied with the change of color from blue to colourless (as shown in Figure S8).

\section{CONCLUSIONS}

We have successfully synthesized $\mathrm{ZnO} / \mathrm{C}$ nanocomposites by a simple one-step high temperature heat treatment of MOF-5 in different carbonization atmospheres. The $\mathrm{ZnO} / \mathrm{C}$ composites with different carbon/metal oxide ratios are formed when derived at $800^{\circ} \mathrm{C}$ and $1000{ }^{\circ} \mathrm{C}$ in air, argon and water vapor atmospheres respectively. After the carbonization process the inherent porosity and cubic structure of precursor MOF-5 remain in the resulting composites. All the composites demonstrate high photocatalytic activity compared to the pure $\mathrm{ZnO}$ nanoparticles obtained by heat treatment of MOF-5 in air. The composite derived at $1000^{\circ} \mathrm{C}$ in argon saturated with water vapors for a short duration $(1 \mathrm{~h})$ outperforms all the other composites regarding the photocatalytic MB degradation. Defects in the crystalline structure of carbon doped $\mathrm{ZnO}$ and the presence of oxygen-containing hydrophilic $-\mathrm{COOH}$ functional groups on the surface of the composite functioned as traps for dye molecules and facilitate their photodegradation. The present study provides a simple and promising approach for the synthesis and application of homogeneously dispersed porous $\mathrm{ZnO} / \mathrm{C}$ composites with excellent photocatalytic performance for the removal of organic pollutants from water.

\section{ASSOCIATED CONTENT}

\section{Supporting Information}

Supplementary data related to this article can be found in file attached. 


\section{ACKNOWLEDGEMENTS}

The authors are thankful to EPSRC CDT in Metamaterials at University of Exeter for financial support (EP/L015331/1). AS acknowledges the Deutsche Forschungsgemeinschaft (German Research Council) for a postdoctoral fellowship. The authors also thank to Dr. Jayaramulu Kolleboyina for his contribution to design the representative figure.

\section{Present Addresses}

c Sandia National Laboratories, Livermore, CA 94551-0696 (USA)

\section{REFERENCES}

(1) Rochkind M.; Pasternak S.; Paz. Y. Using Dyes for Evaluating Photocatalytic Properties: A Critical Review. Molecules 2015, 20, 88-110.

(2) Kubacka A.; Fernandez-Garcia M.; Colon G. Advanced Nanoarchitectures for Solar Photocatalytic Applications. Chem. Rev. 2011, 112, 1555-1614.

(3) Galian R. E.; Perez-Prieto P. Catalytic Processes Activated by Light. Energy Environ. Sci., 2010, 3, 1488-1498.

(4) Wang J.; Wang Z.; Huang B.; Ma Y.; Liu Y.; Qin X.; Zhang X.; Dai Y. Oxygen Vacancy Induced Band-Gap Narrowing and Enhanced Visible Light Photocatalytic Activity of $\mathrm{ZnO}$. Appl. Mater. Interfaces 2012, 4, 4024-430.

(5) Liu S.; Li C.; Yu J.; Xiang Q. Improved Visible-Light Photocatalytic Activity of Porous Carbon Self-Doped Zno Nanosheet-Assembled Flowers. CrystEngComm. 2011, 13, 25332541.

(6) Cho S.; Jang J.; Lee J.; Lee K., Carbon-Doped Zno Nanostructures Synthesized Using Vitamin C for Visible Light Photocatalysis. CrystEngComm. 2010, 12, 3929-3935.

(7) Alshammari A. S.; Chi L.; Chen X.; Bagabas A.; Kramer D.; Alromaeha A.; Jiang Z. VisibleLight Photocatalysis on C-Doped ZnO Derived from Polymer-Assisted Pyrolysis. RSC Adv. 2015, 5, 27690-27698. 
(8) Oar-Arteta L.; Wezendonk T.; Sun X.; Kapteijn F.; Gascon J. Metal Organic Frameworks as Precursors for the Manufacture of Advanced Catalytic Materials. Mater. Chem. Front. 2017, $1,1709-1745$.

(9) Yang S. J.; Nam S.; Kim T.; Im J. H.; Jung H.; Kang J. H.; Wi S.; Park B.; Park C. R. Preparation and Exceptional Lithium Anodic Performance of Porous Carbon-Coated ZnO Quantum Dots Derived from a Metal-Organic Framework. J. Am. Chem. Soc. 2013, 135, 7394-7397.

(10) Pan L.; Muhammad T.; Ma L.; Huang Z.; Wang S.; Wang L.; Zou J.; Zhang X. MOFDerived C-Doped ZnO Prepared via a Two-Step Calcination for Efficient Photocatalysis. Appl. Catal. B: Environ. 2016, 189, 181-191.

(11) Sun J.; Xu Q. Functional Materials Derived from Open Framework Templates/Precursors: Synthesis and Applications. Energy Environ. Sci. 2014, 7, 2071-2100.

(12) Tranchemontagne D. J.; Hunt J. R.; Yaghi O. M. Room Temperature Synthesis of MetalOrganic Frameworks: MOF-5, MOF-74, MOF-177, MOF-199, and IRMOF-0. Tetrahedron 2008, 64, 8553-8557.

(13) Yaghi O. M.; O’Keeffe M.; Ockwig N. W.; Chae H. K.; Eddaoudi M.; Kim J. Reticular Synthesis and the Design of New Materials. Nature 2003, 423, 705-714.

(14) Li H.; Eddaoudi M.; O'Keeffe M.; Yaghi O. M. Design and Synthesis of an Exceptionally Stable and Highly Porous Metal-Organic Framework. Nature 1999, 402, 276-279.

(15) Wu H.; Gong Q.; Olson D. H.; Li J. Commensurate Adsorption of Hydrocarbons and Alcohols in Microporous Metal Organic Frameworks. Chem. Rev. 2012, 112, 836-868.

(16) Banerjee R.; Furukawa H.; Britt D.; Knobler C.; O’Keeffe M.; Yaghi O. M. Control of Pore Size and Functionality in Isoreticular Zeolitic Imidazolate Frameworks and their Carbon Dioxide Selective Capture Properties. J. Am. Chem. Soc. 2009, 131, 3875-3877.

(17) Horcajada P.; Gref R.; Baati T.; Allan P. K.; Maurin G.; Couvreur P.; Ferey G.; Morris R. E.; Serre C. Metal-Organic Frameworks in Biomedicine. Chem. Rev. 2012, 112, 1232-1268. 
(18) Sheberla D.; Bachman J. C.; Elias J. S.; Sun C.; Shao-Horn Y.; Dincă M. Conductive MOF Electrodes for Stable Supercapacitors with High Areal Capacitance. Nature Mater. 2017, 16, 220-224.

(19) Demir-Cakan R.; Morcrette M.; Nouar F.; Davoisne C.; Devic T.; Gonbeau D.; Dominko R.; Serre C.; F'erey G.; Tarascon J. M. Cathode Composites for Li-S Batteries via the Use of Oxygenated Porous Architectures. J. Am. Chem. Soc. 2011, 133, 16154-16160.

(20) Yoon M.; Srirambalaji R.; Kim K. Homochiral Metal-Organic Frameworks for Asymmetric Heterogeneous Catalysis. Chem. Rev. 2012, 112, 1196-1231.

(21) Kreissl H. T.; Li M. M. J.; Peng Y.; Nakagawa K.; Hooper T. J. N.; Hanna J. V.; Shepherd A.; Wu T.; Soo Y.; Tsang S. C. E. Structural Studies of Bulk to Nanosize Niobium Oxides with Correlation to Their Acidity. J. Am. Chem. Soc. 2017, 139, 12670-12680.

(22) Kaye S. S.; Dailly A.; Yaghi O. M.; Long J. R. Impact of Preparation and Handling on the Hydrogen Storage Properties of $\mathrm{Zn}_{4} \mathrm{O}(1,4-$ benzenedicarboxylate) 3 (MOF-5). J. Am. Chem. Soc. 2007, 129, 14176-14177.

(23) Xie Z.; Xu w.; Cui X.; Wang Y. Recent Progress in Metal-Organic Frameworks and Their Derived Nanostructures for Energy and Environmental Applications. ChemSusChem 2017, $10,1645-1663$.

(24) Chen Y.; Zhang R.; Jiao L.; Jiang H. Metal-Organic Framework-Derived Porous Materials for Catalysis. Coord. Chem. Rev. 2018, 362, 1-23

(25) Teng W.; Bai N.; Chen Z.; Shi J.; Fan J.; Zhang W. Hierarchically Porous Carbon Derived from Metal-Organic Frameworks for Separation of Aromatic Pollutants. Chem. Eng. J. 2018, 346, 388-396.

(26) Yang S. J.; Im J. H.; Kim T.; Lee K.; Park C. R. MOF-Derived ZnO and Zno@C Composites with High Photocatalytic Activity and Adsorption Capacity. J. Hazard. Mater. 2011, 186, 376-382.

(27) Chen B.; Ma G.; Kong D.; Zhu Y.; Y. Xia Y. Atomically Homogeneous Dispersed ZnO/NDoped Nanoporous Carbon Composites with Enhanced $\mathrm{CO}_{2}$ Uptake Capacities and High Efficient Organic Pollutants Removal from Water. Carbon 2015, 95, 113-124. 
(28) Ma M.; Zacher D.; Zhang X.; Fischer R. A.; Metzler-Nolte N. A Method for the Preparation of Highly Porous, Nanosized Crystals of Isoreticular Metal-Organic Frameworks. Cryst. Growth DesigN 2011, 11, 185-189.

(29) Huang L.; Wang H.; Chen J.; Wang Z.; Sun J.; Zhao D.; Yan Y. Synthesis, Morphology Control, and Properties of Porous Metal-Organic Coordination Polymers. Micropor. Mesopor. Mater. 2003, 58, 105-114.

(30) Abbasi Z.; Shamsaei E.; Leong S. K.; Ladewig B.; Zhang X.; Wang H. Effect of Carbonization Temperature on Adsorption Property of ZIF-8 Derived Nanoporous Carbon for Water Treatment. Micropor. Mesopor. Mater. 2016, 236, 28-37.

(31) Hafizovic J.; Bjørgen M.; Olsbye U.; Dietzel P. D. C.; Bordiga S.; Prestipino C.; Lamberti C.; Lillerud K. P. The Inconsistency in Adsorption Properties and Powder XRD Data of MOF-5 is Rationalized by Framework Interpenetration and the Presence of Organic and Inorganic Species in the Nanocavities. J. Am. Chem. Soc. 2007, 129, 3612-3620.

(32) Nesakumar N.; Rayappan J. B. B.; Gopalakrishnan B.; Prakash J.; Krishnan U. M. Influence of $\mathrm{pH}$ on Structural Morphology of ZnO Nanoparticle. J. Appl.Sci. 2012, 12, 1758-1768.

(33) Torad N. L.; Hu M.; Ishihara S.; Sukegawa H.; Belik A. A.; Imura M.; Ariga K.; Sakka Y.; Yamauchi Y. Direct Synthesis of MOF-derived Nanoporous Carbon With Magnetic Co Nanoparticles Toward Efficient Water Treatment. Small 2014, 10, 2096-2107.

(34) Srinivas G.; Krungleviciute V.; Guo Z.; T. Yildirim T. Exceptional $\mathrm{CO}_{2}$ Capture in A Hierarchically Porous Carbon With Simultaneous High Surface Area and Pore Volume. Energy Environ. Sci. 2014, 7, 335-342.

(35) Han M.; Yin X.; Ren S.; Duan W.; Zhang L.; Cheng L. Core/shell Structured C/ZnO Nanoparticles Composites for Effective Electromagnetic Wave Absorption. RSC Adv. 2016, 6, 6467-6474.

(36) Özgür Ü.; Alivov Y. I.; Liu C.; Teke A.; Reshchikov M. A.; Doğan S.; Avrutin V.; Cho S.J.; Morkoç H. A Comprehensive Review of ZnO Materials and Devices. J. Appl. Phys. 2005, 98, 041301. 
(37) Son D. I.; Kwon B. W.; Park D. H.; Seo W.; Yi Y.; Angadi B.; Lee C.; Choi W. K. Emissive ZnO-graphene Quantum Dots for White-Light-Emitting Diodes. Nature Nanotechno. 2012, 7, 465-471.

(38) Cuscó R.; Alarcón-Lladó E.; Ibáñez J.; Artus L.; Jimenez J.; Wang B.; Callahan M. J. Temperature Dependence of Raman Scattering in ZnO. Phys. Rev. B 2007, 75, 165202.

(39) Chu P. K.; Li L. Characterization of Amorphous and Nanocrystalline Carbon Films. Mater. Chem. Phys. 2006, 96, 253-277.

(40) Ferrari A. C.; Robertson J. Interpretation of Raman Spectra of Disordered and Amorphous Carbon. Phys. Rev. B 2000, 61, 14095-14107.

(41) Dillon R. O.; Woollam J. A.; Katkanant V. Use of Raman Scattering to Investigate Disorder and Crystallite Formation in as-deposited and Annealed Carbon Films. Phys. Rev. B 1984, $29,3482-3489$.

(42) Tai F. C.; Lee S. C.; Wei C. H.; Tyan S. L. Correlation between ID/AG Ratio from Visible Raman Spectra and sp2/sp3 Ratio from XPS Spectra of Annealed Hydrogenated DLC Film. Mater. Trans. 2006, 47, 1847-1852.

(43) Thakur S.; Barua S.; Karak N. Reduced Graphene Oxide-Metal Oxide Nanohybrid for Efficient Adsorption, Photodegradation and Photoinactivation of Chemical and Microbial Contaminants. J. Nanotech. Diagnosis Treatment 2015, 3, 12-22.

(44) Mishra D.K.; Mohapatra J.; Sharma M. K.; Chattarjee R.; Singh S. K.; Varma S.; S.N. Behera S.N.; Nayak S. K.; Entel P. Carbon Doped ZnO: Synthesis, Characterization and Interpretation. J. Magn. Magn. Mater. 2013, 329, 146-152.

(45) Yang S.; Zhi L.; Tang K.; Feng X. L.; Maier J.; Mullen K. Efficient Synthesis of Heteroatom (N or S)-Doped Graphene Based on Ultrathin Graphene Oxide-Porous Silica Sheets for Oxygen Reduction Reactions. Adv. Funct. Mater. 2012, 22, 3634-3641.

(46) Wang J.; Zhong H. X.; Qin Y. L.; Zhang X. B. An Efficient Three-Dimensional Oxygen Evolution Electrode. Angew. Chem. Int. Ed. 2013, 52, 5248-5253.

(47) Ferrari A. C. Raman Spectroscopy of Graphene and Graphite: Disorder, Electron-Phonon Coupling, Doping and Nonadiabatic Effects. Solid State Commun. 2007, 143, 1-2, 47-57. 
(48) Liang P.; Zhang C.; Sun H.; Liu S.; Tade M.; Wang S. Solar Photocatalytic Water Oxidation and Purification on ZIF-8-Derived C-N-ZnO Composites. Energy Fuels 2017, 31, 21382143. 